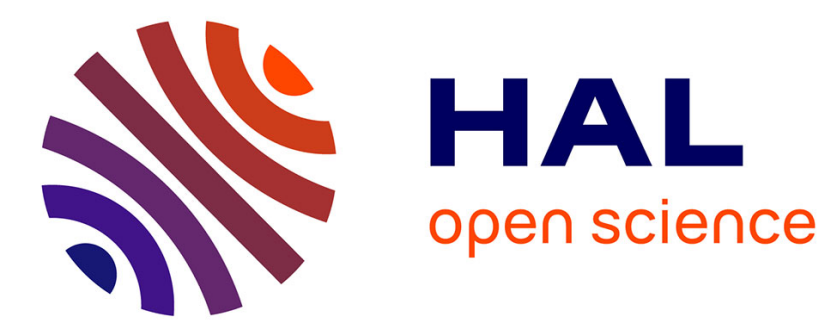

\title{
Oscillatory Kelvin-Helmholtz instability. Part 1. A viscous theory
}

Harunori Yoshikawa, José Eduardo Wesfreid

\section{To cite this version:}

Harunori Yoshikawa, José Eduardo Wesfreid. Oscillatory Kelvin-Helmholtz instability. Part 1. A viscous theory. Journal of Fluid Mechanics, 2011, 675, pp.223-248. 10.1017/S0022112011000140 . hal-00906510

\section{HAL Id: hal-00906510 https://hal.science/hal-00906510}

Submitted on 19 Nov 2013

HAL is a multi-disciplinary open access archive for the deposit and dissemination of scientific research documents, whether they are published or not. The documents may come from teaching and research institutions in France or abroad, or from public or private research centers.
L'archive ouverte pluridisciplinaire HAL, est destinée au dépôt et à la diffusion de documents scientifiques de niveau recherche, publiés ou non, émanant des établissements d'enseignement et de recherche français ou étrangers, des laboratoires publics ou privés. 


\title{
Oscillatory Kelvin-Helmholtz instability: Part 1. A viscous theory
}

\author{
HARUNORI N. YOSHIKA WA $\dagger$ \\ andJ OSÉ EDUARDO WESFREID \\ Physique et Mécanique des Milieux Hétérogènes (PMMH) \\ UMR 7636 CNRS - ESPCI - UPMC Univ. Paris 06 - PDD Univ. Paris 07 \\ 10, rue Vauquelin 75231 Paris Cedex 5, France
}

(Received ?? and in revised form ??)

The stability of oscillatory two-layer flows is investigated with a linear perturbation analysis. An asymptotic case is considered where the oscillation amplitude is small compared to the perturbation wavelength. The focus of the analysis is on the influence of viscosity and its contrast at the interface. The flows are unstable when the relative velocity of the layers is larger than a critical value. Depending on the oscillation frequency, the flows are in different dynamical regimes, which are characterized by the relative importance between capillary wavelength and the thicknesses of the Stokes boundary layers developed on the interface. A particular regime is found in which instability occurs at a substantially lower critical velocity. The mechanism behind the instability is studied by identifying the velocity- and shear-induced components in the disturbance growth rate. They interchange dominance depending on the frequency and the viscosity contrast. Results of the analysis are compared with experiments in the literature. Good agreement is found with the experiments that have a small oscillation amplitude. The validity condition of the asymptotic theory is estimated.

Key words: Boundary layer stability; Multiphase flow; Pattern formation

\section{Introduction}

The stability of the interface between fluid layers subjected to parametric excitation has attracted research interest (Davis 1976). A well-known example of such a system is a fluid layer that is vertically vibrated. Faraday instability can develop standing waves on their interface (Miles \& Henderson 1990). In inviscid approximation, the linear stability of this system is analyzed by the Mathieu equation (Benjamin \& Ursell 1954). Harmonic and subharmonic parametric resonances are expected to occur. A linear stability analysis for viscous fluids shows a damping of higher resonance modes (Kumar \& Tuckerman 1994). Although many studies have been devoted to Faraday instability, an analogous problem with horizontal vibration has been studied less.

Horizontal vibrations applied to a container of two superposed fluid layers generates a horizontal pressure gradient that induces oscillatory shear flows. In the inviscid approximation, the stability of this flow is analyzed by the Mathieu equation (Kelly 1965). However, an additional unstable mode should be taken into account in addition to the

$\dagger$ Present address: Laboratoire J.-A. Dieudonné, Université de Nice Sophia-Antipolis, Parc Valrose - 06108 Nice Cedex 2, France. 
parametric resonances. This mode is not a standing wave; it results in interface waves oscillating in the horizontal direction. The marginal stability curve of this mode was derived for flows with a small oscillation amplitude in thick layers of an identical thickness (Lyubimov \& Cherepanov 1987):

$$
\frac{\rho_{1} \rho_{2}}{2\left(\rho_{1}+\rho_{2}\right)} \Delta U^{2}=\frac{2 \pi \gamma}{\lambda}+\frac{\left(\rho_{1}-\rho_{2}\right) g}{2 \pi / \lambda}
$$

where $\rho_{1}$ and $\rho_{2}$ are the densities of the lower and upper fluids, flowing with a velocity $U_{1 \max } \cos (\omega t)$ and $U_{2 \max } \cos (\omega t)$, respectively. The velocity difference $\Delta U$ is defined by $\Delta U=U_{2 \max }-U_{1 \max }$. The density difference, $\rho_{1}-\rho_{2}$, is supposed to be positive. Unstable superposition of fluids will not be considered in the present paper. $\gamma$ is the interfacial tension, $g$ is gravitational acceleration, and $\lambda$ is the wavelength. The phase velocity $c_{0}$ of the growing waves is identical to the velocity of the centre of mass:

$$
c_{o}=\frac{\rho_{1} U_{1 \max }+\rho_{2} U_{2 \max }}{\rho_{1}+\rho_{2}} \cos (\omega t)
$$

Results (1.1) and (1.2) are the same as those of the classical Kelvin-Helmholtz instability, when $\Delta U / \sqrt{2}$ is regarded as the velocity difference in the classical case. The influence of the finite layer thicknesses (Lyubimov \& Cherepanov 1987; Lyubimov et al. 2003) and finite oscillation amplitude (Yoshikawa 2006) on this Kelvin-Helmholtz (KH) mode instability in the oscillatory flows was also examined.

Viscous flows are considered in the case of a thin liquid layer on a horizontal plate. Linear stability analyses were performed with use of Floquet theory for plate oscillation with a small oscillation amplitude compared to the wavelength (Yih 1967) and with a finite amplitude (Or 1997). Wave formation was found beyond an instability onset. Superposed thin layers shearing each other were also considered with the use of similar mathematical methods (Coward \& Papageorgiou 1994; King et al. 1999).

The linear stability of flows in thick fluid layers was investigated for long waves, i.e., waves of $\lambda$ much longer than the layer thicknesses (Kamachi \& Honji 1982). Waves with a finite wavelength were considered with numerical resolutions of the stability problem for a small viscosity contrast (Khenner et al. 1999) and for a large viscosity contrast (Talib \& Juel 2007). A strong viscous damping on parametric resonance modes was found. The KH mode instability was found to be hardly influenced by the viscosity for fluid layers with a small viscosity contrast, while a substantial decrease of the instability threshold was found in fluid layers with a large viscosity contrast. Linear stability analysis for fluids of infinite depth also showed a substantial threshold decrease for fluids with a large viscosity contrast within a certain oscillation frequency range (Yoshikawa 2006). This frequency range is associated with a certain relationship between characteristic length scales, as shown in the present paper (see section 4).

Experiments were performed with thin and thick liquid layers. All the experiments concerned only an instability leading to the formation of oscillating waves. To the authors' knowledge, no observation of parametric resonances has been reported. King et al. (1999) investigated the stability of thin layers. They confined two density-matched fluids in the small gap of two concentric cylinders. The interface of the fluids was in the middle of the gap and parallel to the cylinder walls. Fluids were sheared by oscillatory rotation of the outer cylinder wall. They found wavy patterns on the interface perpendicular to the motion.

Shyh \& Munson (1986) used a vertically installed cylindrical tank, in which two thick fluid layers with very different viscosities were superposed. They drove flows by turning 


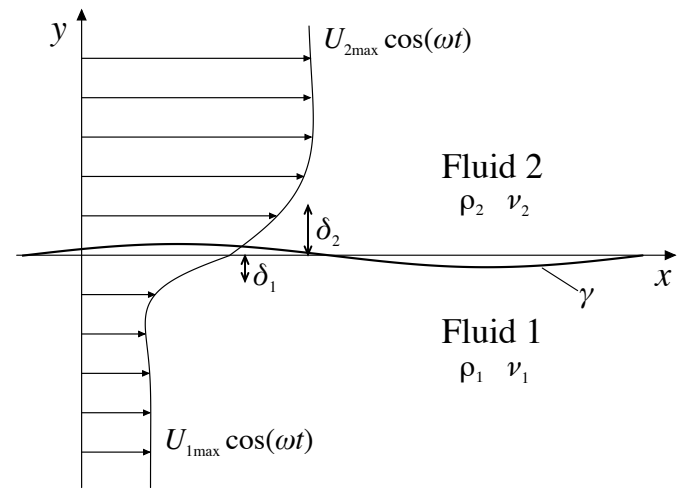

Figure 1. Oscillatory flow in semi-infinite fluid layers. Lower fluid is assumed to be less viscous throughout the paper $\left(\nu_{1} \leqslant \nu_{2}\right)$.

it periodically around its centre axis. The layers were then in oscillatory relative motion in the azimuthal direction. They found formation of waves perpendicular to the motion. In our experimental paper which is coupled with the present one (Yoshikawa \& Wesfreid 2010), our setup utilized the same principle.

Wolf (1969), Beysens et al. (1998), Ivanova et al. (2001a) and Talib et al. (2007) used the horizontal vibration of a fluid container to drive flows with a horizontal pressure gradient. Cylindrical and parallelepiped containers were used. Similar experiments with miscible fluids were performed in a parallelepiped container by Legendre et al. (2003). Ivanova et al. $(2001 b)$ applied a circularly polarized vibration in the horizontal plane to a cylindrical container. The horizontal oscillatory motion of observed waves is typically small. These waves look static and are often referred to as "frozen waves."

Although experimentally observed patterns seem to result from the KH mode instability, the inviscid theory (1.1) was found to be unable to predict the interface behaviour correctly (Beysens et al. 1998; Ivanova et al. 2001a,b). In experiments, the critical velocity and wavelength of the patterns depended significantly on the oscillation frequency, while they are constant according to the theory.

The present paper aims to clarify the influence of viscosity and its contrast at the interface on the KH mode instability. Insights into the underlying mechanism will also be achieved. We consider flows in superposed semi-infinite layers with a linear perturbation analysis. This configuration is wall-free and enables us to study essential features of the instability.

The dynamics of an interface disturbance of wavelength $\lambda$ is decided by the physical properties of fluids $\left(\rho_{1}, \rho_{2}, \gamma\right.$ and viscosities $\nu_{1}$ and $\nu_{2}$, where we will assume throughout the paper that the lower fluid is less viscous, $\nu_{1} \leqslant \nu_{2}$ ), the relative velocity $\Delta U$, the frequency $\omega / 2 \pi$, and the gravitational acceleration $g$ (see figure 1). After the Buckingham $\Pi$ theorem, the dynamics depend on only six dimensionless parameters. They can be the density and viscosity contrasts $\rho=\rho_{2} / \rho_{1}$ and $\kappa=\sqrt{\nu_{2} / \nu_{1}}(\geqslant 1)$, a dimensionless amplitude $K=2 \omega^{-1} \Delta U / \lambda$, a dimensionless frequency $\Omega=\lambda_{\text {cap }}^{2} \omega / 2 \nu_{2}\left(\lambda_{\text {cap }}\right.$ is the capillary wavelength: $\left.\lambda_{c a p}=2 \pi \sqrt{\gamma /\left(\rho_{1}-\rho_{2}\right) g}\right)$, a dimensionless wavenumber $q=\lambda_{\text {cap }} / \lambda$ and the parameter $B$, which is hereafter referred to as the velocity parameter:

$$
\mathrm{B}=\frac{\pi \rho_{1} \rho_{2}}{2\left(\rho_{1}^{2}-\rho_{2}^{2}\right)} \frac{\Delta U^{2}}{g \lambda_{c a p}}
$$




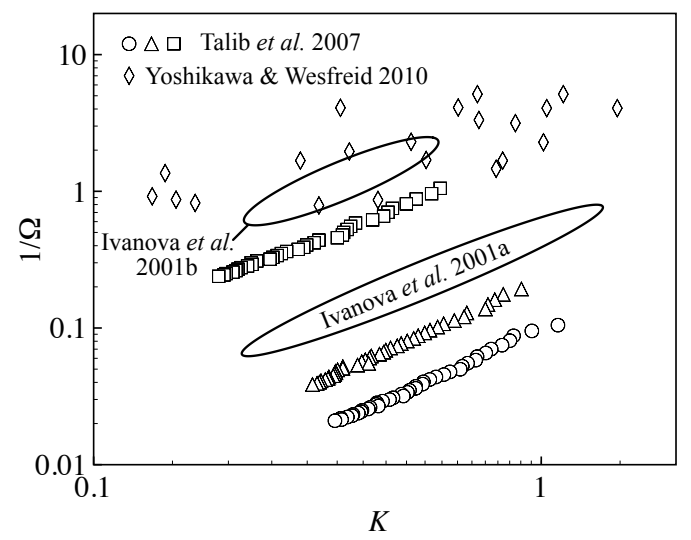

Figure 2. Parameter ranges of existing experiments. The inviscid theory for small amplitude oscillatory flows (1.1) is valid in the limit of $K \rightarrow 0$ and $1 / \Omega \rightarrow 0$.

Any other possible dimensionless groups can be expressed by these six parameters. As seen in sections 4 and 5, our choice is convenient for distinguishing different dynamical regimes in the instability and to compare the viscous theory with the inviscid one.

The velocity parameter compares hydrodynamic pressures to capillary pressures exerted at the interface. We will regard it as a primary control parameter. The coefficient in equation (1.3), composed of the densities, has been multiplied in order to transform the marginal curve (1.1) into its simplest form:

$$
\mathrm{B}=\frac{q+q^{-1}}{2}
$$

The corresponding instability threshold $\mathrm{B}_{c r}$ and critical wavenumber $q_{c r}$ are given by:

$$
\mathrm{B}_{c r}=1, \quad q_{c r}=1
$$

The dimensionless amplitude $K$ is twice the ratio of the oscillation amplitude $\Delta U / \omega$ to the wavelength and is identical to the Keulegan-Carpenter number. This number characterizes oscillatory flows over a curved boundary, comparing the advection $(\boldsymbol{u} \cdot \boldsymbol{\nabla})$ with the local variation $(\partial / \partial t)$ (see, e.g., Rousseaux et al. 2004). In a typical experiment with thick layers, the wavelength $\lambda$ is on the order of $\lambda_{\text {cap }}(\approx 5 \mathrm{~mm})$ and the amplitude varies from $0.5 \mathrm{~mm}$ to $3 \mathrm{~mm}$. The value of $K$ is typically between 0.3 and 10 . The dimensionless frequency $\Omega$ can be regarded as a square-ratio of $\lambda_{\text {cap }}$ to the Stokes boundary layer thickness $\delta_{2}=\sqrt{2 \nu_{2} / \omega}$ in the more viscous fluid: $\Omega=\left(\lambda_{\text {cap }} / \delta_{2}\right)^{2}$. As seen in section 2.1, the Stokes boundary layers develop in both fluids on the interface and their thicknesses give unique length scales that are involved in the velocity profiles of the basic flows. In section 4 , it will be shown that the relationships of these length scales with $\lambda_{c a p}$ characterize different regimes in the two-layer flows. In experiments, the oscillation frequency varies typically from $10 \mathrm{~Hz}$ to $50 \mathrm{~Hz}$ and the values of $\delta_{2}$ are between $1 \mathrm{~mm}$ and $6 \mathrm{~mm}$. The typical range of $\Omega$ is between 1 and 20 .

The theoretical result (1.1), or equivalently (1.4), was derived using inviscid approximation with the assumption of a small oscillation amplitude relative to the wavelength. These hypotheses correspond to the limits $\Omega^{-1} \rightarrow 0$ and $K \rightarrow 0$. The discrepancies with the experiments reported in the literature are due to the breaking of these hypotheses. Indeed, the values of $\Omega^{-1}$ and $K$ are not always small in the experiments. Nevertheless, one of these parameters typically takes a small value. Figure 2 shows the range of these parameters as examined in the experimental works. Most of the experiments were per- 
formed either with small $\Omega^{-1}$ or small $K$. The asymptotic consideration for the cases $\Omega^{-1} \ll 1$ and $K \ll 1$ are worthwhile in predicting the stability under practical conditions. In the present paper, we will tackle the linear stability problem by asymptotic expansion about a small $K$.

The basic flow for perturbation analysis is presented in the next section. The linear stability problem is then formulated in the dimensionless form. In nondimensionalization, all the lengths involved in a fluid layer are scaled by the thickness of the Stokes boundary layer developed on the interface in order to capture the dynamics inside this layer. This is in contrast with the preceding viscous theories, where the thickness of the layers was chosen as the scaling length. The KH mode instability, however, has an interface origin and the latter scaling seems to hide a clear understanding of the instability. The formulated problem is solved in section 3 by the asymptotic expansion of about $K=0$. Results are presented in section 4 with a focus on the viscosity influence. In section 5 , wave motion and time-averaged flow patterns are discussed to characterize different dynamical regimes of oscillatory two-layer flows. Examination of different origins of perturbation flow is also performed to bring insights into the mechanism of the instability. The validity of the theory is shown through comparison with the existing theoretical and experimental results. Conclusions are given in section 6 .

\section{Mathematical formulation}

Semi-infinite fluid layers are considered (see figure 1). The lower fluid, Fluid 1, is assumed to be less viscous than the second fluid, Fluid 2, like most of the experiments in the literature. The fluids are stably stratified with an immiscible interface at $y=0$. Imposing oscillatory flows $\boldsymbol{u}_{j}=U_{j \max } \cos (\omega t) \hat{\mathbf{x}}(j=1,2 ; \hat{\mathbf{x}}$ : the unit vector in the horizontal direction) far from the interface in each fluid, we first determine the basic flow established on a plane interface. After discussing different flow configurations in which this basic flow can arise, we formulate the linear stability problem.

\subsection{Basic flow}

One-dimensional velocity fields $\boldsymbol{u}_{j}=U_{j}(y, t) \hat{\mathbf{x}}$ in Fluid $j(j=1,2)$ are determined by the Navier-Stokes equations:

$$
\rho_{1} \frac{\partial U_{1}}{\partial t}=-\frac{\partial P_{1}}{\partial x}+\rho_{1} \nu_{1} \frac{\partial^{2} U_{1}}{\partial y^{2}}, \quad 0=-\frac{\partial P_{1}}{\partial y}-\rho_{1} g
$$

for $y<0$, and

$$
\rho_{2} \frac{\partial U_{2}}{\partial t}=-\frac{\partial P_{2}}{\partial x}+\rho_{2} \nu_{2} \frac{\partial^{2} U_{2}}{\partial y^{2}}, \quad 0=-\frac{\partial P_{2}}{\partial y}-\rho_{2} g
$$

for $y>0 . P_{j}(j=1,2)$ stands for the pressure field in Fluid $j$. The velocities match the imposed velocities far from the interface:

$$
\begin{array}{ccc}
U_{1} \rightarrow U_{1 \max } \cos (\omega t) & \text { as } & y \rightarrow-\infty \\
U_{2} \rightarrow U_{2 \max } \cos (\omega t) & \text { as } & y \rightarrow \infty
\end{array}
$$

and obey the velocity continuity and the stress balance at the interface $(y=0)$ :

$$
U_{1}=U_{2}, \quad \rho_{1} \nu_{1} \frac{\partial U_{1}}{\partial y}=\rho_{2} \nu_{2} \frac{\partial U_{2}}{\partial y}, \quad P_{1}=P_{2}
$$

The basic flow is the time-periodic solution of these equations. They are:

$$
U_{1}=U_{\mathrm{i}}(t)+W_{1}(t, y)
$$




$$
U_{2}=U_{\mathrm{i}}(t)+W_{2}(t, y)
$$

with

$$
\begin{aligned}
& W_{1}=-\frac{\rho \kappa \Delta U}{1+\rho \kappa}\left[\cos (\omega t)-e^{\frac{y}{\delta_{1}}} \cos \left(\omega t+\frac{y}{\delta_{1}}\right)\right] \\
& W_{2}=\frac{\Delta U}{1+\rho \kappa}\left[\cos (\omega t)-e^{-\frac{y}{\delta_{2}}} \cos \left(\omega t-\frac{y}{\delta_{2}}\right)\right]
\end{aligned}
$$

where $\delta_{1}$ is the Stokes boundary layer thickness in Fluid 1: $\delta_{1}=\sqrt{2 \nu_{1} / \omega}$. The other component $U_{\mathrm{i}}(t)$ is the velocity at the interface level $(y=0)$. It is given by:

$$
U_{\mathrm{i}}=\frac{U_{1 \max }+\rho \kappa U_{2 \max }}{1+\rho \kappa} \cos (\omega t)
$$

The above basic flow can arise in different experimental configurations. Among those of importance are the flow in a horizontally vibrated container, the duct flow generated by a pulsating pressure gradient and the oscillatory Couette flow (Talib \& Juel (2007) called the first configuration "counterflowing layers." The third configuration includes the case of "co-flowing layers" considered in Talib (2006)). Under certain conditions, these flows are identical to the flow $(2.5-2.8)$ in the neighbourhood of the interface, with different $U_{\mathrm{i}}$ and $\Delta U$ in different flow configurations (for more details, see appendix $\mathrm{A}$ ). When both fluid layers are thick, i.e., $H_{1} \gg \delta_{1}$ and $H_{2} \gg \delta_{2}\left(H_{1}\right.$ and $H_{2}$ are the thicknesses of Fluid 1 and Fluid 2, respectively), the flow in a horizontally vibrated container is given by equations (2.5-2.8) near the interface, with

$$
U_{\mathrm{i}}=\frac{\rho(1+\kappa)\left(H_{1}+H_{2}\right)}{(1+\rho \kappa)\left(\rho H_{1}+H_{2}\right)} U_{\text {cont }} \cos \omega t \quad \text { and } \quad \Delta U=\frac{(1-\rho)\left(H_{1}+H_{2}\right)}{\rho H_{1}+H_{2}} U_{\text {cont }}
$$

where $U_{\text {cont }}$ is the container velocity. This velocity difference $\Delta U$ agrees with the result obtained by Khenner et al. (1999) in inviscid approximation. Fluid layers are thick in many practical situations, e.g., a $10 \mathrm{~mm}$ layer of a fluid of viscosity $10 \mathrm{~mm}^{2} / \mathrm{s}$ will be thick for a frequency higher than $1 \mathrm{~Hz}$ (the Stokes boundary layer thickness is $1.8 \mathrm{~mm}$ ). The flow generated by a pulsating pressure difference, $\Delta P \sin \omega t$ per unit length, is also given by equations (2.5-2.8) near the interface, when both layers are thick. The velocities $U_{\mathrm{i}}$ and $\Delta U$ for this flow are:

$$
U_{\mathrm{i}}=\frac{\rho(1+\kappa) \Delta P}{(1+\rho \kappa) \rho_{2} \omega} \cos \omega t \quad \text { and } \quad \Delta U=\frac{(1-\rho) \Delta P}{\rho_{2} \omega}
$$

The Couette flow is induced by the horizontal oscillatory motion of the bottom and top walls bounding the fluids. When the less viscous layer (Fluid 1 ) is thick: $H_{1} \gg \delta_{1}$, and the more viscous layer (Fluid 2) is thin: $H_{2} \ll \delta_{2}$, the Couette flow near the interface is given by $U_{1}=U_{2 \text {,wall }} e^{\frac{y}{\delta_{1}}} \cos \left(\omega t+\frac{y}{\delta_{1}}\right)$ and $U_{2}=U_{2 \text {,wall }} \cos \omega t$, where $U_{2 \text {, wall }}$ is the velocity of the wall bounding Fluid 2. This flow is identical to the basic flow (2.5-2.8) in the limit of large viscosity contrast $(\kappa \gg 1)$.

Another realisation of the basic flow (2.5-2.8) is the flow in a vertical cylindrical tank rotated alternatively around its centre axis, as the experiments of Shyh \& Munson (1986) and Yoshikawa \& Wesfreid (2010). Motion of the tank wall induces an oscillatory flow in the azimuthal direction. A complete analysis of this flow is presented in Yoshikawa \& Wesfreid (2010). According to the analysis, if the less viscous layer is thick and the viscosity contrast is large, the flow near the interface is given by equations (2.5-2.8) with radially varying $U_{\mathrm{i}}$ and $\Delta U$, except in a layer of thickness around $5 \delta_{1}$ on the tank wall. The KH mode instability concerns the maximum $\Delta U$. It occurs near the wall and is given 
by the following equation for a large tank radius $R\left(\gg \delta_{1}\right)$ :

$$
\Delta U=R \omega \Phi_{0}
$$

where $\Phi_{0}$ is the rotation amplitude of the tank in radian. Centrifugal effects have been neglected in the analysis. This assumption will be valid, as long as $0.79 \nu_{1} / R \ll R \omega \Phi_{0} \ll$ $\sqrt{8\left(\rho_{1}-\rho_{2}\right) g R / \rho_{1}}$ (for more details, see Yoshikawa \& Wesfreid 2010).

The stability of these different flows can therefore be investigated by considering the basic flow (2.5-2.8) with using relevant $U_{\mathrm{i}}$ and $\Delta U$. We will formulate the linear stability problem of this basic flow in section 2.2. As seen in equations (2.5-2.8), the velocity field in Fluid $j(j=1,2)$ varies significantly over the Stokes boundary layer developed on the interface. Its thickness $\delta_{j}$ is a unique length scale involved in the velocity profile. To take into account the influence of the flow inside this layer, we will adopt $\delta_{j}$ as the length scale in the nondimensionalization of the linear stability problem. It is also seen that the velocity profiles in both fluids are similar to each other in the interface reference frame, i.e., the reference frame moving at the velocity $U_{\mathrm{i}} \hat{\mathbf{x}}$. When the viscosity contrast is large $(\kappa \gg 1)$, it moves with the more viscous layer (see equation $(2.9)$ ). At the other limit $(\kappa=1)$, it moves with the centre of mass at velocity (1.2). We will formulate the problem in this reference frame to simplify the mathematical treatments with the similarity.

\subsection{Perturbation flows}

A small interface disturbance $\eta$ and its associated perturbation flow are analyzed by linearized vorticity equations and boundary conditions. To describe the flow in Fluid 1 and Fluid 2, we use stream functions $\psi_{1}$ and $\psi_{2}$, respectively. They are related to the perturbation velocity field $\boldsymbol{u}_{j}$ in Fluid $j(j=1,2)$ by $\boldsymbol{u}_{j}=\boldsymbol{\nabla} \times\left(\psi_{j} \hat{\mathbf{z}}\right)$. The governing equations are partial differential equations with coefficients varying periodically with time. The stability governed by such equations can be determined using Floquet theory (Yih 1967). The interface disturbance and stream functions of wave length $\lambda$ are then decomposed into their net exponential growth $e^{\sigma t}$ and time-periodic variations:

$$
\begin{aligned}
\eta & =e^{i \frac{2 \pi}{\lambda} x} e^{\sigma t} \sum_{m} \widetilde{\eta}_{m} e^{i m \omega t}+c . c . \\
\psi_{1} & =e^{i \frac{2 \pi}{\lambda} x} e^{\sigma t} \sum_{m} \widetilde{\psi}_{1, m}(y) e^{i m \omega t}+c . c . \\
\psi_{2} & =e^{i \frac{2 \pi}{\lambda} x} e^{\sigma t} \sum_{m} \widetilde{\psi}_{2, m}(y) e^{i m \omega t}+c . c .
\end{aligned}
$$

where c.c. denotes the complex conjugate of its preceding term. These decompositions are substituted in the linearized governing equations to formulate a linear stability problem.

For nondimensionalization, we adopt the time scale $\omega^{-1}$ and the velocity scale $\Delta U$. Lengths in Fluid 1 and Fluid 2 are scaled by $\delta_{1}$ and $\delta_{2}$, respectively, for the reason explained earlier. Due to different length scales in different fluids, the dimensionless spatial coordinates are denoted as $x_{j}$ and $y_{j}$ in Fluid $j(j=1,2)$. The governing equations, decomposed with use of (2.13-2.15), are written in dimensionless form:

Vorticity equation in Fluid $1\left(y_{1}<0\right)$

$$
\left(\triangle_{1}-2 \sigma-i 2 m\right) \triangle_{1} \widetilde{\psi}_{1, m}=i 2 \pi K\left[\left(\widetilde{W}_{1,1} \triangle_{1}-\widetilde{W}_{1,1}^{\prime \prime}\right) \widetilde{\psi}_{1, m-1}+\left(\widetilde{W}_{1,-1} \triangle_{1}-\widetilde{W}_{1,-1}^{\prime \prime}\right) \widetilde{\psi}_{1, m+1}\right]
$$


Vorticity equation in Fluid $2\left(y_{2}>0\right)$

$\left(\triangle_{2}-2 \sigma-i 2 m\right) \triangle_{2} \widetilde{\psi}_{2, m}=i 2 \pi K\left[\left(\widetilde{W}_{2,1} \triangle_{2}-\widetilde{W}_{2,1}^{\prime \prime}\right) \widetilde{\psi}_{2, m-1}+\left(\widetilde{W}_{2,-1} \triangle_{2}-\widetilde{W}_{2,-1}^{\prime \prime}\right) \widetilde{\psi}_{2, m+1}\right]$

Perturbation decay far away from the interface

$$
\widetilde{\psi}_{1, m} \rightarrow 0 \quad \text { as } \quad y_{1} \rightarrow-\infty, \quad \widetilde{\psi}_{2, m} \rightarrow 0 \quad \text { as } \quad y_{2} \rightarrow \infty
$$

Velocity continuity (tangent to the interface)

$$
\widetilde{\psi}_{1, m}^{\prime}-\widetilde{\psi}_{2, m}^{\prime}+\frac{\rho \kappa^{2}-1}{\rho \kappa^{2}}\left(\widetilde{W}_{1,1}^{\prime} \widetilde{\eta}_{m-1}+\widetilde{W}_{1,-1}^{\prime} \widetilde{\eta}_{m+1}\right)=0 \quad \text { at } y=0
$$

Velocity continuity (normal to the interface)

$$
\widetilde{\psi}_{1, m}-\kappa \widetilde{\psi}_{2, m}=0 \quad \text { at } y=0
$$

Stress balance (tangent to the interface)

$$
\begin{gathered}
\widetilde{\psi}_{1, m}^{\prime \prime}+\frac{4 \pi^{2} q^{2}}{\kappa^{2} \Omega} \widetilde{\psi}_{1, m}-\rho \kappa\left(\widetilde{\psi}_{2, m}^{\prime \prime}+\frac{4 \pi^{2} q^{2}}{\Omega} \widetilde{\psi}_{2, m}\right)+\frac{\kappa+1}{\kappa}\left(\widetilde{W}_{1,1}^{\prime \prime} \widetilde{\eta}_{m-1}+\widetilde{W}_{1,-1}^{\prime \prime} \widetilde{\eta}_{m+1}\right)=0 \\
\text { at } y=0
\end{gathered}
$$

Stress balance (normal to the interface)

$$
\begin{gathered}
\widetilde{\psi}_{1, m}^{\prime \prime \prime}-\left(\frac{12 \pi^{2} q^{2}}{\kappa^{2} \Omega}+2 \sigma+i 2 m\right) \widetilde{\psi}_{1, m}^{\prime}+i 2 \pi K\left(\widetilde{W}_{1,1}^{\prime} \widetilde{\psi}_{1, m-1}+\widetilde{W}_{1,-1}^{\prime} \widetilde{\psi}_{1, m+1}\right) \\
-\rho\left[\widetilde{\psi}_{2, m}^{\prime \prime \prime}-\left(\frac{12 \pi^{2} q^{2}}{\Omega}+2 \sigma+i 2 m\right) \widetilde{\psi}_{2, m}^{\prime}+i 2 \pi K\left(\widetilde{W}_{2,1}^{\prime} \widetilde{\psi}_{2, m-1}+\widetilde{W}_{2,-1}^{\prime} \widetilde{\psi}_{2, m+1}\right)\right] \\
-i \frac{\pi^{2} \rho K\left(1+q^{2}\right)}{\kappa(1+\rho) \mathrm{B} \sqrt{\Omega}} \widetilde{\eta}_{m}=0 \quad \text { at } y=0,
\end{gathered}
$$

Kinematic equation of a material interface

$$
i \pi K \widetilde{\psi}_{1, m}+(\sigma+i m) \widetilde{\eta}_{m}=0 \quad \text { at } y=0
$$

for the mode $m(=0, \pm 1, \pm 2, \ldots)$. The basic velocities $W_{j}(j=1,2)$ are decomposed into frequency components: $W_{j}=\widetilde{W}_{j, 1} e^{i t}+\widetilde{W}_{j,-1} e^{-i t}$. A prime on a function associated with Fluid $j(j=1,2)$ means differentiation with respect to $y_{j}$. The operators $\triangle_{1}$ and $\triangle_{2}$ are two-dimensional Laplacians:

$$
\triangle_{1}=\frac{d^{2}}{d y_{1}^{2}}-\frac{4 \pi^{2} q^{2}}{\kappa^{2} \Omega}, \quad \triangle_{2}=\frac{d^{2}}{d y_{2}^{2}}-\frac{4 \pi^{2} q^{2}}{\Omega}
$$

In the above formulation, dimensionless groups resulting from nondimensionalization have been transformed into expressions in terms of the dimensionless parameters introduced in section 1 . The terms $\frac{4 \pi^{2} q^{2}}{\kappa^{2} \Omega}$ and $\frac{4 \pi^{2} q^{2}}{\Omega}$ in (2.24) are, for example, the squares of the dimensionless wavenumbers $\frac{2 \pi \delta_{1}}{\lambda}$ and $\frac{2 \pi \delta_{2}}{\lambda}$, which appear naturally during nondimensionalization. The last term in the left-hand-side of equation (2.22) represents contributions from gravity and capillarity. It is written, in dimensional form, $(4 \pi / \lambda)\left[\left(\rho_{1}-\rho_{2}\right) g+\right.$ $\left.\gamma(2 \pi / \lambda)^{2}\right] \widetilde{\eta}_{m} / \rho_{1} \omega \Delta U$.

The equation set (2.16-2.23) defines a linear stability problem for arbitrary values of the dimensionless parameters, $\rho, \kappa, q, \mathrm{~B}, \Omega$ and $K$. In the next section, we will solve this problem for the asymptotic case of $K \ll 1$. 


\section{Asymptotic consideration for small oscillation amplitude flows}

We perform formal expansions of $\widetilde{\eta}_{m}, \widetilde{\psi}_{j, m}(j=1,2)$ and $\sigma$ about a small parameter $2 \pi K$ :

$$
\begin{aligned}
\widetilde{\eta}_{m} & =\hat{\eta}_{m}^{(0)}+2 \pi K \hat{\eta}_{m}^{(1)}+(2 \pi K)^{2} \hat{\eta}_{m}^{(2)}+\cdots \\
\widetilde{\psi}_{j, m} & =\hat{\psi}_{j, m}^{(0)}+2 \pi K \hat{\psi}_{j, m}^{(1)}+(2 \pi K)^{2} \hat{\psi}_{j, m}^{(2)}+\cdots \\
\sigma & =\sigma^{(0)}+2 \pi K \sigma^{(1)}+(2 \pi K)^{2} \sigma^{(2)}+\cdots
\end{aligned}
$$

Inserting these expansions into equations (2.16-2.23), we separate the stability problem according to the order with respect to $K$ for a resolution, order by order.

\subsection{First order problem}

On the first order, i.e., $O\left(K^{0}\right)$-order, the kinematic equation (2.23) is reduced to:

$$
\left(\sigma^{(0)}+i m\right) \hat{\eta}_{m}^{(0)}=0 \quad(m=0, \pm 1, \pm 2, \cdots)
$$

It follows that the interface wave has no unsteady component:

$$
\hat{\eta}_{m}^{(0)}=0 \text { for } m= \pm 1, \pm 2, \cdots
$$

For the steady mode, equation (3.4) implies that $\sigma^{(0)}=0$ or $\hat{\eta}_{0}^{(0)}=0$. The latter case means no interface disturbance and is inconsistent with the Kelvin-Helmholtz instability scenario: small interface disturbances grow through dynamical positive feedback. We will therefore consider only the former case and set

$$
\hat{\eta}_{0}^{(0)}=1 \quad \text { and } \quad \hat{\eta}_{0}^{(n)}=0 \quad \text { for } n=1,2, \cdots
$$

without any loss of generality.

The vorticity equations (2.16) and (2.17) and the boundary conditions (2.18-2.22) are reduced to:

$$
\begin{aligned}
& \left(\triangle_{1}-i 2 m\right) \triangle_{1} \hat{\psi}_{1, m}^{(0)}=0 \\
& \left(\triangle_{2}-i 2 m\right) \triangle_{2} \hat{\psi}_{2, m}^{(0)}=0 \\
& \left\{\begin{array}{ccc}
\hat{\psi}_{1, m}^{(0)} \rightarrow 0 & \text { as } & y_{1} \rightarrow-\infty \\
\hat{\psi}_{2, m}^{(0)} \rightarrow 0 & \text { as } & y_{2} \rightarrow \infty
\end{array}\right. \\
& \hat{\psi}_{1, m}^{\prime(0)}-\hat{\psi}_{2, m}^{\prime(0)}=-\frac{\rho \kappa^{2}-1}{\rho \kappa^{2}}\left(\widetilde{W}_{1,1}^{\prime} \hat{\eta}_{m-1}^{(0)}+\widetilde{W}_{1,-1}^{\prime} \hat{\eta}_{m+1}^{(0)}\right) \\
& \hat{\psi}_{1, m}^{(0)}-\kappa \hat{\psi}_{2, m}^{(0)}=0 \\
& \hat{\psi}_{1, m}^{\prime \prime(0)}+\frac{4 \pi^{2} q^{2}}{\kappa^{2} \Omega} \hat{\psi}_{1, m}^{(0)}-\rho \kappa\left(\hat{\psi}_{2, m}^{\prime \prime(0)}+\frac{4 \pi^{2} q^{2}}{\Omega} \hat{\psi}_{2, m}^{(0)}\right)=-\frac{\kappa+1}{\kappa}\left(\widetilde{W}_{1,1}^{\prime \prime} \hat{\eta}_{m-1}^{(0)}+\widetilde{W}_{1,-1}^{\prime \prime} \hat{\eta}_{m+1}^{(0)}\right) \\
& \hat{\psi}_{1, m}^{\prime \prime \prime(0)}-\left(\frac{12 \pi^{2} q^{2}}{\kappa^{2} \Omega}+i 2 m\right) \hat{\psi}_{1, m}^{\prime(0)}-\rho\left[\hat{\psi}_{2, m}^{\prime \prime \prime}(0)-\left(\frac{12 \pi^{2} q^{2}}{\Omega}+i 2 m\right) \hat{\psi}_{2, m}^{\prime(0)}\right]=0
\end{aligned}
$$

for the mode $m(=0, \pm 1, \pm 2, \cdots)$. Equations (3.10-3.13) are boundary conditions at the interface $\left(y_{1}=y_{2}=0\right)$. These equation sets are homogeneous for any mode $m$, except for the fundamental unsteady modes $m= \pm 1$, as the interface wave has no unsteady component (see equation (3.5)). It follows that

$$
\hat{\psi}_{1, m}^{(0)}=0, \quad \hat{\psi}_{2, m}^{(0)}=0 \quad \text { for } m=0, \pm 2, \pm 3, \cdots
$$


For the mode $m=1$, the solutions of the vorticity equations (3.7) and (3.8) with the boundary conditions (3.9) are:

$$
\begin{gathered}
\hat{\psi}_{1,1}^{(0)}=a_{1,1}^{(0)} e^{\frac{2 \pi q}{\kappa \sqrt{\Omega}} y_{1}}+b_{1,1}^{(0)} e^{\sqrt{\frac{4 \pi^{2} q^{2}}{\kappa^{2} \Omega}+i 2} y_{1}} \\
\hat{\psi}_{2,1}^{(0)}=a_{2,1}^{(0)} e^{-\frac{2 \pi q}{\sqrt{\Omega}} y_{2}}+b_{2,1}^{(0)} e^{-\sqrt{\frac{4 \pi^{2} q^{2}}{\Omega}+i 2} y_{2}}
\end{gathered}
$$

The constants, $a_{1,1}^{(0)}, b_{1,1}^{(0)}, a_{2,1}^{(0)}$ and $b_{2,1}^{(0)}$, are determined by the linear algebraic equation set, which is obtained by substituting (3.15) into the boundary conditions (3.10-3.13). The equation set writes:

$$
\boldsymbol{L}_{1}\left[a_{1,1}^{(0)} b_{1,1}^{(0)} a_{2,1}^{(0)} b_{2,1}^{(0)}\right]^{t r}=\boldsymbol{d}_{1}^{(0)}
$$

The subscript 1 on the matrix $\boldsymbol{L}$ and the vector $\boldsymbol{d}$ means they concern the mode $m=1$. Explicit definitions of $\boldsymbol{L}_{1}$ and $\boldsymbol{d}_{1}^{(0)}$ are given in appendix B. The flow of the other mode $m=-1$ is determined by the symmetry of the considered system. It requires $\hat{\psi}_{1,-1}^{(0)}=\hat{\psi}_{1,1}^{*(0)}$ and $\hat{\psi}_{2,-1}^{(0)}=\hat{\psi}_{2,1}^{*(0)}$, where the asterisks indicate a complex conjugate (see appendix $\mathrm{C}$ ).

The second order growth rate, $\sigma^{(1)}$, is determined by the first order flow. The kinematic equation (2.23), reduced at the second order, writes

$$
\frac{1}{2} \hat{\psi}_{1, m}^{(0)}-i \sigma^{(1)} \hat{\eta}_{m}^{(0)}+m \hat{\eta}_{m}^{(1)}=0
$$

With use of equation (3.14) this gives, for the steady mode,

$$
\sigma^{(1)}=-\frac{i}{2} \hat{\psi}_{1,0}^{(0)}(0)=0
$$

The system is neutrally stable up to the second order, as expected. Indeed, the first order flow $\hat{\psi}_{1,0}^{(0)}$ is the solution in the Stokes limit, as $K=0$ means that there is no inertial term in vorticity equations. No net evolution of the system is possible due to the reversibility of Stokes flows. The growth rate (3.18), given by the first order flow, should therefore be equal to zero.

From equation (3.17), the second order interface disturbance is also deduced:

$$
\hat{\eta}_{1}^{(1)}=-\frac{\hat{\psi}_{1,1}^{(0)}}{2}, \quad \hat{\eta}_{-1}^{(1)}=\frac{\hat{\psi}_{1,-1}^{(0)}}{2} \quad \text { and } \quad \hat{\eta}_{m}^{(1)}=0 \quad(m=0, \pm 2, \pm 3, \cdots)
$$

In order to determine the stability, the third order growth rate $\sigma^{(2)}$ should be calculated, as shown in the second order problem below. The results of the above consideration are used there as inputs.

\subsection{Second order problem}

In section 3.1, we saw that the growth rate $\sigma^{(1)}$ is decided by the steady component of the previous order flow. This is also the case for $\sigma^{(2)}$, as we will see below. We therefore consider only the steady components of flow on the second order, i.e., $O(K)$-order.

Using the previous results (3.18) and (3.19), the vorticity equations (2.16) and (2.17) and the boundary conditions $(2.18-2.22)$ for the steady mode are reduced to the following:

$$
\begin{aligned}
\triangle_{1}^{2} \hat{\psi}_{1,0}^{(1)} & =i\left[\widetilde{W}_{1,-1} \triangle_{1}-\widetilde{W}_{1,-1}^{\prime \prime}\right] \hat{\psi}_{1,1}^{(0)}-\text { c.c. } \\
\triangle_{2}^{2} \hat{\psi}_{2,0}^{(1)} & =i\left[\widetilde{W}_{2,-1} \triangle_{2}-\widetilde{W}_{2,-1}^{\prime \prime}\right] \hat{\psi}_{2,1}^{(0)}-\text { c.c. } \\
& \hat{\psi}_{1,0}^{(1)} \rightarrow 0 \quad \text { as } \quad y_{1} \rightarrow-\infty \\
& \hat{\psi}_{2,0}^{(1)} \rightarrow 0 \quad \text { as } \quad y_{2} \rightarrow \infty
\end{aligned}
$$




$$
\begin{gathered}
\hat{\psi}_{1,0}^{\prime(1)}-\hat{\psi}_{2,0}^{\prime(1)}=\frac{\rho \kappa^{2}-1}{2 \rho \kappa^{2}}\left(\widetilde{W}_{1,-1}^{\prime} \hat{\psi}_{1,1}^{(0)}-c . c .\right) \\
\hat{\psi}_{1,0}^{(1)}-\kappa \hat{\psi}_{2,0}^{(1)}=0 \\
\hat{\psi}_{1,0}^{\prime \prime(1)}+\frac{4 \pi^{2} q^{2}}{\kappa^{2} \Omega} \hat{\psi}_{1,0}^{(1)}-\rho \kappa\left(\hat{\psi}_{2,0}^{\prime \prime(1)}+\frac{4 \pi^{2} q^{2}}{\Omega} \hat{\psi}_{2,0}^{(1)}\right)=\frac{\kappa+1}{2 \kappa}\left(\widetilde{W}_{1,-1}^{\prime \prime} \hat{\psi}_{1,1}^{(0)}-\text { c.c. }\right) \\
\hat{\psi}_{1,0}^{\prime \prime \prime}(1)-\frac{12 \pi^{2} q^{2}}{\kappa^{2} \Omega} \hat{\psi}_{1,0}^{(1)}-\rho\left(\hat{\psi}_{2,0}^{\prime \prime \prime}(1)-\frac{12 \pi^{2} q^{2}}{\Omega} \hat{\psi}_{2,0}^{\prime(1)}\right)= \\
-\left(i \widetilde{W}_{1,-1}^{\prime} \hat{\psi}_{1,1}^{(0)}-\text { c.c. }\right)+\rho\left(i \widetilde{W}_{2,-1}^{\prime} \hat{\psi}_{2,1}^{(0)}-\text { c.c. }\right)-\frac{i \pi \rho\left(1+q^{2}\right)}{2(1+\rho) \kappa \mathrm{B} \sqrt{\Omega}}
\end{gathered}
$$

Equations (3.23-3.26) are boundary conditions at the interface $\left(y_{1}=y_{2}=0\right)$. The right-hand-sides of (3.20) and (3.21) are the advections associated with the $m= \pm 1$ components of the first order flow. The terms c.c. are contributions from the flow of $m=-1$. (Remember that $\psi_{1,1}^{*(0)}=\psi_{1,-1}^{(0)}$ and $\psi_{2,1}^{*(0)}=\psi_{2,-1}^{(0)}$, due to symmetry.)

The vorticity equations (3.20) and (3.21) and the boundary conditions (3.22) require solutions in the following form:

$$
\begin{aligned}
& \hat{\psi}_{1,0}^{(1)}=a_{1,0}^{(1)} e^{\frac{2 \pi q}{\kappa \sqrt{\Omega}} y_{1}}+b_{1,0}^{(1)} \frac{2 \pi q}{\kappa \sqrt{\Omega}} y_{1} e^{\frac{2 \pi q}{\kappa \sqrt{\Omega}} y_{1}}+\varphi_{1}\left(y_{1}\right) \\
& \hat{\psi}_{2,0}^{(1)}=a_{2,0}^{(1)} e^{-\frac{2 \pi q}{\sqrt{\Omega}} y_{2}}+b_{2,0}^{(1)} \frac{2 \pi q}{\sqrt{\Omega}} y_{2} e^{-\frac{2 \pi q}{\sqrt{\Omega}} y_{2}}+\varphi_{2}\left(y_{2}\right)
\end{aligned}
$$

where $\varphi_{1}\left(y_{1}\right)$ and $\varphi_{2}\left(y_{1}\right)$ are particular solutions reflecting the advection terms. They are

$$
\begin{gathered}
\varphi_{1}=\left[F_{1} a_{1,1}^{(0)} e^{\left(\frac{2 \pi q}{\kappa \sqrt{\Omega}}+1-i\right) y_{1}}+G_{1} b_{1,1}^{(0)} e^{\sqrt{\frac{4 \pi^{2} q^{2}}{\kappa^{2} \Omega}+i 2} y_{1}}\right. \\
\left.+H_{1} b_{1,1}^{(0)} e^{\left(\sqrt{\frac{4 \pi^{2} q^{2}}{\kappa^{2} \Omega}+i 2}+1-i\right) y_{1}}\right]-c . c . \\
\varphi_{2}=\left[F_{2} a_{2,1}^{(0)} e^{-\left(\frac{2 \pi q}{\sqrt{\Omega}}+1-i\right) y_{2}}+G_{2} b_{2,1}^{(0)} e^{-\sqrt{\frac{4 \pi^{2} q^{2}}{\Omega}+i 2} y_{2}}\right. \\
\left.+H_{2} b_{2,1}^{(0)} e^{-\left(\sqrt{\frac{4 \pi^{2} q^{2}}{\Omega}+i 2}+1-i\right) y_{2}}\right]-c . c .
\end{gathered}
$$

The coefficients $F_{j}, G_{j}$ and $H_{j}(j=1,2)$ can be derived from the substitution of these equations in equations (3.20) and (3.21). They are given in appendix D.

Inserting equation (3.27) into the boundary conditions (3.23-3.26), one will find a linear algebraic equation set with respect to the constants $a_{1,0}^{(1)}, b_{1,0}^{(1)}, a_{2,0}^{(1)}$ and $b_{2,0}^{(1)}$. It writes:

$$
\boldsymbol{L}_{0}\left[a_{1,0}^{(1)} b_{1,0}^{(1)} a_{2,0}^{(1)} b_{2,0}^{(1)}\right]^{t r}=\boldsymbol{d}_{0}^{(1)}
$$

The subscript 0 on the matrix $\boldsymbol{L}$ and on the vector $\boldsymbol{d}$ means that they concern the steady mode $m=0$. The vector $\boldsymbol{d}_{0}^{(1)}$ is a function of the first order flow. Explicit definitions of $\boldsymbol{L}_{0}$ and $\boldsymbol{d}_{0}^{(1)}$ are given in appendix B.

The third order growth rate, $\sigma^{(2)}$, is decided through the kinematic equation on the third order for the steady mode: $i \hat{\psi}_{1,0}^{(1)}+2 \sigma^{(2)}=0$. With use of $(3.27)$ and (3.28), it follows that

$$
\sigma^{(2)}=-\frac{i \hat{\psi}_{1,0}^{(1)}(0)}{2}=-\frac{i}{2}\left[a_{1,0}^{(1)}+\left(F_{1} a_{1,1}^{(0)}+G_{1} b_{1,1}^{(0)}+H_{1} b_{1,1}^{(0)}\right)-c . c .\right]
$$




\begin{tabular}{|c|c|c|c|}
\hline Regime & Marginal curve & Critical values & Validity condition \\
\hline $\begin{array}{l}\text { (i) High frequency } \\
\text { (ii) Moderate frequency } \\
\text { (iii) Low frequency }\end{array}$ & $\begin{array}{c}\mathrm{B}=\frac{q^{-1}+q}{2} \\
\mathrm{~B}=\frac{\rho}{1+\rho} \frac{q^{-1}+q}{2} \\
\text { (no instability) }\end{array}$ & $\begin{array}{c}\mathrm{B}_{c r}=1, q_{c r}=1 \\
\mathrm{~B}_{c r}=\frac{\rho}{1+\rho}, q_{c r}=1 \\
\text { (no instability) }\end{array}$ & $\begin{array}{c}\Omega>10^{4} \\
\frac{560}{\kappa^{2}}<\Omega<10 \rho, \kappa>100 \\
\Omega \ll \frac{q^{2}}{\kappa^{2}}\end{array}$ \\
\hline
\end{tabular}

This predicts a net growth $\left(\sigma^{(2)}>0\right)$ and decay $\left(\sigma^{(2)}<0\right)$ of the disturbance. In the next section, we will investigate the stability by computing $\sigma^{(2)}$ using this equation.

\section{Stability}

Calculations necessary to obtain the growth rate $\sigma^{(2)}$ by (3.31) are the inversions of the linear equation sets (3.16) and (3.30). In general, these are performed numerically. In particular cases with certain assumptions about the frequency $\Omega$, analytical calculations are carried out. Simple analytical dispersion relations are derived. We first consider the particular cases and then proceed to the general consideration. The particular cases are (i) the high frequency regime where $\Omega \gg q^{2}$, (ii) the moderate frequency regime where $\frac{q^{2}}{\kappa^{2}} \ll \Omega \ll q^{2}$ and (iii) the low frequency regime where $\Omega \ll \frac{q^{2}}{\kappa^{2}}$. In dimensional terms, these relationships are equivalent to (i) $\delta_{2} \ll \lambda$, (ii) $\delta_{1} \ll \lambda \ll \delta_{2}$, and (iii) $\lambda \ll \delta_{1}$, respectively. Note that case (ii) is possible only in fluid layers with a large viscosity contrast, i.e., $\kappa \gg 1$. It should also be noted that the relationships $\delta_{1} \ll \lambda$ and $\lambda \ll \delta_{2}$ are implied in case (i) and case (ii), respectively. Fluid 2 is always assumed to be more viscous than Fluid $1\left(\nu_{1} \leqslant \nu_{2}\right)$. The results obtained below for these cases are summarized in table 1.

\subsection{High frequency regime: $\Omega \gg q^{2}$}

We describe only the outline of the derivation of the dispersion relation because algebraic procedures to calculate $\sigma^{(2)}$ are straightforward. The matrix $\boldsymbol{L}_{1}$ in equation (3.16) includes the small parameter $\epsilon_{a}=\frac{2 \pi q}{\sqrt{\Omega}}$. The inverse matrix, determined by Cramer's formula, can be expanded in a power series of $\epsilon_{a}: \boldsymbol{L}_{1}^{-1}=\epsilon_{a}^{-1} \boldsymbol{l}^{(-1)}+\boldsymbol{l}^{(0)}+O\left(\epsilon_{a}\right)$. The first order coefficients are given by $\left[a_{1,1}^{(0)} b_{1,1}^{(0)} a_{2,1}^{(0)} b_{2,1}^{(0)}\right]^{t r}=\boldsymbol{l}^{(0)} \boldsymbol{d}^{(0)}+O\left(\epsilon_{a}\right)$ (the product $\boldsymbol{l}^{(-1)} \boldsymbol{d}^{(0)}$ yields a zero-vector). These results are used to calculate $\boldsymbol{d}_{0}^{(1)}$ in the second order problem. The inverse matrix of $\boldsymbol{L}_{0}$ is also expanded similarly: $\boldsymbol{L}_{0}^{-1}=\epsilon_{a}^{-3} \boldsymbol{m}^{(-3)}+\epsilon_{a}^{-2} \boldsymbol{m}^{(-2)}+O\left(\epsilon_{a}^{-1}\right)$ and is used to calculate the second order coefficients, $a_{1,0}^{(1)}, b_{1,0}^{(1)}, a_{2,0}^{(1)}$ and $b_{2,0}^{(1)}$. Finally, injection of these results into equation (3.31) yields the dispersion relation:

$$
\sigma^{(2)}=\epsilon_{a}^{-2} \frac{\rho \kappa^{2}}{16(1+\rho)\left(1+\rho \kappa^{2}\right)}\left(1-\frac{q^{-1}+q}{2 \mathrm{~B}}\right)+O\left(\epsilon_{a}^{-1}\right)
$$

The corresponding marginal curve, instability threshold, $\mathrm{B}_{c r}$, and critical wavenumber, $q_{c r}$, are given in table 1 . This recovers the result of the inviscid theory (1.4). This suggests that, for the inviscid approximation, the hypothesis made for the present regime should be satisfied, i.e., $\Omega \gg q^{2}$. This requirement for the inviscid approximation will be estimated more precisely in section 4.4. 


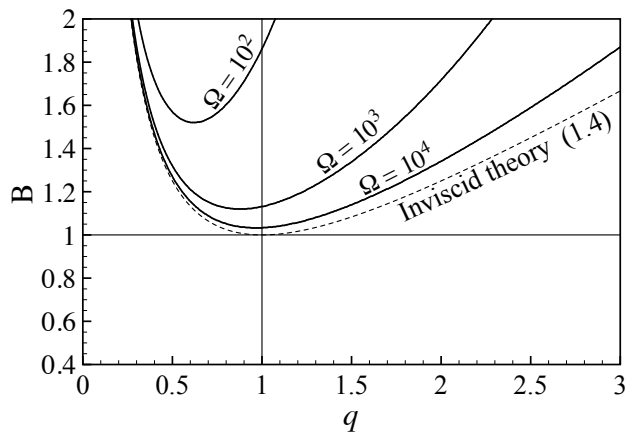

(a) $\kappa=1$

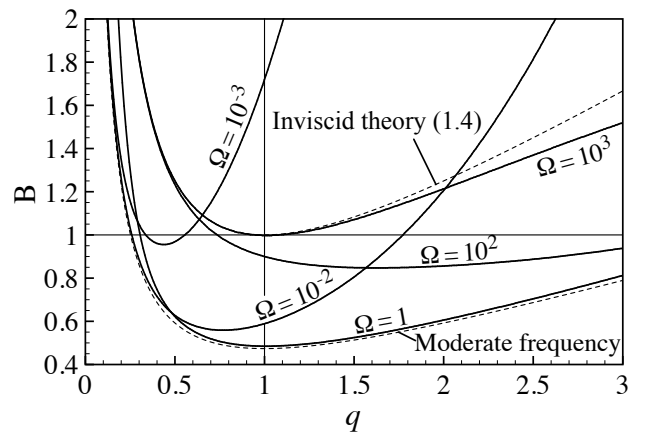

(b) $\kappa=100$

FIGURE 3. Marginal curves for two-layer flows of small and large viscosity contrasts with different oscillation frequency values $\Omega$. The density contrast $\rho$ is 0.9 for all the curves.

\subsection{Moderate frequency regime: $\frac{q^{2}}{\kappa^{2}} \ll \Omega \ll q^{2}$}

The small parameters included in matrices $\boldsymbol{L}_{1}$ and $\boldsymbol{L}_{0}$ are $\epsilon_{b}=\kappa^{-1}$ and $\epsilon_{c}=\frac{\sqrt{\Omega}}{2 \pi q}$ with the relationship $\epsilon_{b} \ll \epsilon_{c}$. The inverse matrices of $\boldsymbol{L}_{1}$ and $\boldsymbol{L}_{0}$ are expanded in power series of $\epsilon_{b}$ and then in power series of $\epsilon_{c}$. The result is $\boldsymbol{L}_{1}^{-1}=\epsilon_{b}^{-2} \boldsymbol{l}^{(-2)}+O\left(\epsilon_{b}^{-1}\right)$ where $\boldsymbol{l}^{(-2)}=\epsilon_{c} \boldsymbol{l}^{(-2,1)}+O\left(\epsilon_{c}^{2}\right)$. Using these expansions as inversions of equations (3.16) and (3.30) and injecting the results into equation (3.31), one will derive the dispersion relation:

$$
\sigma^{(2)}=\epsilon_{c}^{2} \frac{1}{16 \rho}\left(1-\frac{\rho}{1+\rho} \frac{q^{-1}+q}{2 \mathrm{~B}}\right)+O\left(\max \left(\epsilon_{c}^{3}, \epsilon_{b}\right)\right)
$$

The corresponding marginal curve, instability threshold and critical wavenumber are given in table 1 . The instability threshold is characterized by a constant critical value of the control parameter $\mathrm{B}$ and the most unstable mode has the wavenumber $q$, which is equal to unity, as in the inviscid theory.

However, the critical value of $\mathrm{B}$ is smaller by a factor of $\rho /(1+\rho)$. It is interesting to note that the same results were obtained in stability analyses of steady flows with infinitely large viscosity contrast at the interface (Lindsay 1984; Hogan \& Ayyaswamy 1985). The validity condition of the moderate frequency theory will be discussed and estimated more precisely in section 4.4 .

\subsection{Low frequency regime: $\Omega \ll \frac{q^{2}}{\kappa^{2}}$}

The inverse matrices of $\boldsymbol{L}_{1}$ and $\boldsymbol{L}_{0}$ are expanded in power series of $\epsilon_{d}=\frac{\kappa \sqrt{\Omega}}{2 \pi q}: \boldsymbol{L}_{1}^{-1}=\boldsymbol{l}^{(0)}+$ $O\left(\epsilon_{d}\right)$ and $\boldsymbol{L}_{0}^{-1}=\boldsymbol{m}^{(0)}+O\left(\epsilon_{d}\right)$. The growth rate computed under these approximations is always negative:

$$
\sigma^{(2)}=-\epsilon_{d}^{2} \frac{\rho\left(q^{-1}+q\right)}{32(1+\rho)\left(1+\rho \kappa^{2}\right) \mathrm{B}}+O\left(\epsilon_{d}^{3}\right)
$$

Neither marginal stability nor instability is expected. Any waves much shorter than the Stokes boundary layer thicknesses are therefore stable.

\subsection{General case}

Marginal curves obtained by numerical computations of $\sigma^{(2)}$ are shown in figure 3 . The linear equation sets (3.16) and (3.30) are numerically inverted and used as input in (3.31). The velocity parameter $\mathrm{B}$ is varied at a given $q$ to determine the condition giving the neutral stability $\sigma^{(2)}=0$. In (a) and (b), fluids of small and large viscosity contrasts are 


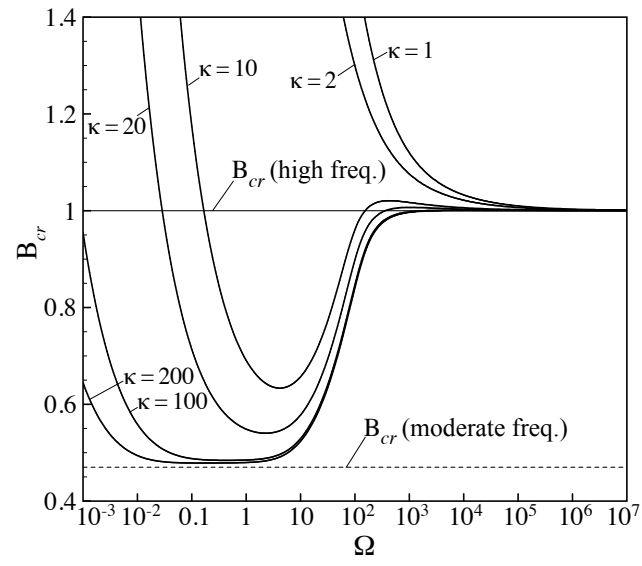

(a) Critical control parameter $\mathrm{B}_{c r}$

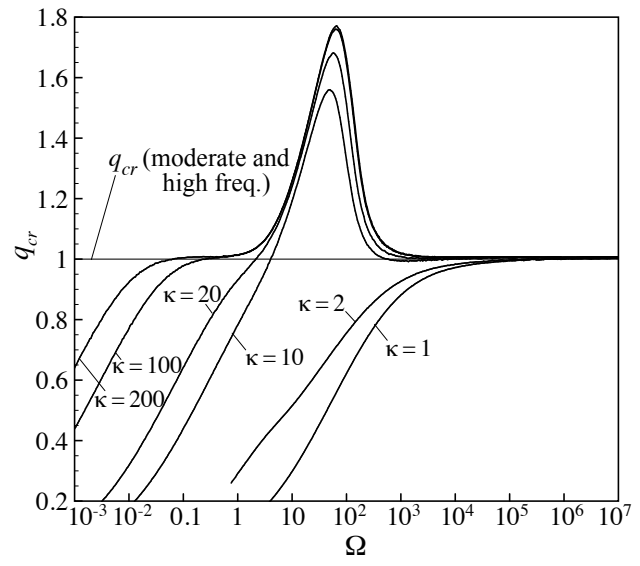

(b) Critical wavenumber $q_{c r}$

Figure 4. Values of the control parameter B and the most unstable mode $q$ on the instability threshold for different viscosity contrast values. The density contrast $\rho$ is 0.9 for all the curves.

considered, respectively. The density contrast is fixed at 0.9. Different curves correspond to different values of the frequency $\Omega$.

All the curves have a minimum $\mathrm{B}_{c r}$ at a finite wavenumber $q=q_{c r}$. When the viscosity contrast is weak, as shown in (a), the minimum $\mathrm{B}_{c r}$ occurs at a $q_{c r}$ smaller than unity. With frequency increasing, the marginal curve descends rightwards and approaches the inviscid curve (1.4). For frequency values larger than $\Omega=10^{5}$, they are almost identical to each other. When the viscosity contrast is large, the behaviour of the marginal curve is more complex. For small frequencies $(\Omega \lesssim 0.1)$, the curve has its minimum at a $q_{c r}$ smaller than unity. With increasing frequency, the curve displaces downwards and approaches the marginal curve of the moderate frequency regime presented in table 1 . After being coincident with the latter, it goes up rightwards and then leftwards, approaching the inviscid marginal curve (1.4).

In figure $4, \mathrm{~B}_{c r}$ and $q_{c r}$ are plotted against $\Omega$ for different values of $\kappa$. The density contrast is fixed at 0.9. For small frequencies, all $\mathrm{B}_{c r}$ curves diverge to infinity. This is consistent with the results for the low frequency regime. When the viscosity contrast is small $(\kappa \sim 1)$, with frequency increasing, $\mathrm{B}_{c r}$ and $q_{c r}$ behave monotonically, approaching unity. For frequencies larger than $10^{4}$, they are equal to unity within a precision of 5 percent, recovering the inviscid results $(1.5)$. When the viscosity contrast is large $(\kappa \gtrsim$ 10 ), with frequency increasing until $\Omega \approx 40 \kappa^{-1}, \mathrm{~B}_{c r}$ decreases towards its minimum value, which is smaller than unity, while $q_{c r}$ increases towards unity. $\mathrm{B}_{c r}$ and $q_{c r}$ stay, respectively, around the minimum value and unity, in the frequency range $560 \kappa^{-2}<$ $\Omega \lesssim 10$. With frequency increasing further, $\mathrm{B}_{c r}$ approaches rapidly to its inviscid value $\left(\mathrm{B}_{c r}=1\right)$, whereas, surprisingly, $q_{c r}$ continues to increase beyond unity and attains its maximum at $\Omega \sim 60$. Then, $q_{c r}$ approaches the inviscid value $\left(q_{c r}=1\right)$. Formation of waves shorter than the capillary wavelength $(q>1)$ was reported in experiments (Talib et al. 2007). Beyond $\Omega=10^{4}$, both $\mathrm{B}_{c r}$ and $q_{c r}$ are almost equal to their asymptotic values and recover the inviscid results. In the case of extremely large viscosity contrast $\left(\kappa>10^{2}\right)$, the frequency range $560 \kappa^{-2}<\Omega<10$ is large, so plateaus are found in the behaviour of $\mathrm{B}_{c r}$ and $q_{c r}$. These plateaus situate at $\mathrm{B}_{c r}=\rho /(1+\rho)$ and $q_{c r}=1$, recovering the earlier analytical results in table 1 for the moderate frequency regime. The behaviour of $\mathrm{B}_{c r}$ and $q_{c r}$ is similar for different values of the density contrast. The upper end of the moderate frequency regime, however, depends on the density contrast as $\sim 10 \rho$. 


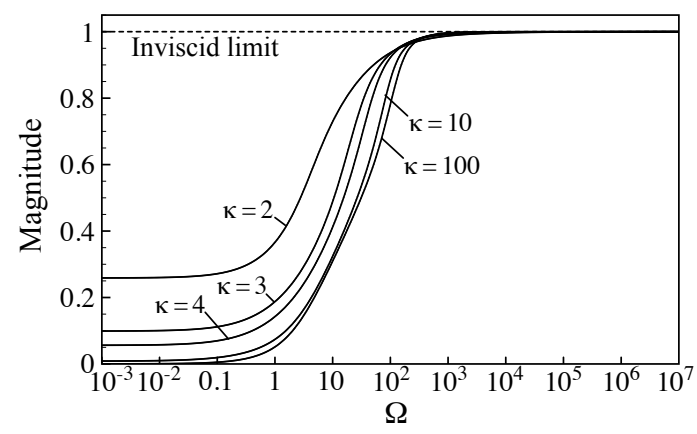

FiguRE 5. Magnitude of the phase velocity of waves in the interface reference frame, at the instability threshold. Magnitude is normalized by the magnitude in the inviscid limit. $(\rho=0.9$ for all the curves.)

This behaviour of $\mathrm{B}_{c r}$ and $q_{c r}$ can be used to determine more precisely the frequency ranges of the high and moderate frequency regimes, i.e., to determine the validity conditions of the results (4.1) and (4.2). The high frequency regime is expected to be a good approximation when $\Omega>10^{4}$ (or in dimensional form, $100 \delta_{2}<\lambda_{\text {cap }}$ ). The moderate frequency regime will be a good approximation when $560 \kappa^{-2}<\Omega<10 \rho$ and $\kappa>100$ (or in dimensional form, $24 \delta_{1}<\lambda_{\text {cap }}<\sqrt{10 \rho} \delta_{2}$ and $100 \delta_{1}<\delta_{2}$ ).

\section{Discussion}

Besides the net growth due to instability, the interface waves oscillate in the horizontal direction. Indeed, the interface disturbance is composed of a steady component $\hat{\eta}_{0}^{(0)}(=1)$ and unsteady components $\hat{\eta}_{ \pm 1}^{(1)}$. The total motion of the interface writes as follows:

$$
\begin{aligned}
\eta & =\left[1+2 \pi K\left(\hat{\eta}_{-1}^{(1)} e^{-i t}+\hat{\eta}_{1}^{(1)} e^{i t}\right)\right] e^{i \frac{2 \pi x}{\lambda}}+c . c . \\
& =2 \cos \left[\frac{2 \pi}{\lambda}\left(x-\frac{1}{\omega} \int c(t) d t\right)\right]
\end{aligned}
$$

for a small $K$. The term $\frac{1}{\omega} \int c d t$ represents oscillatory "propagation" of the waves. The phase velocity $c$ is given by

$$
c=\Delta U\left[-2 \hat{\eta}_{1}^{(1)} e^{i t}+c . c .\right]=\Delta U\left[\left(a_{1,1}^{(0)}+b_{1,1}^{(0)}\right) e^{i t}+c . c .\right]
$$

in dimensional form, where equations (3.15) and (3.19) have been invoked for deriving the last term. Computation of $c$ for different frequencies $\Omega$ and for different viscosity contrasts $\kappa$ shows that the phase velocity is identical to that in the inviscid approximation (1.2) for $\Omega>10^{4}$, indifferently from $\kappa$.

Figure 5 shows the magnitude of $c$ calculated by (5.2) at the instability onset $\left(\mathrm{B}=\mathrm{B}_{c r}\right.$, $\left.q=q_{c r}\right)$. Its value is normalized by the magnitude of the inviscid phase velocity (1.2) in the interface reference frame, i.e., $c_{0}-U_{\mathrm{i}}$. For $\Omega<0.1$, the magnitude is constant for a given $\kappa$. It is equal to zero for $\kappa$ larger than 100. This means that, for such a large $\kappa$, the waves are static in the interface reference frame. Remember that this reference frame moves with Fluid 2 when $\kappa \gg 1$. The waves are hence "frozen" on the more viscous layer, as observed in experiments (Yoshikawa \& Wesfreid 2010). After an increase within a frequency range of $0.1<\Omega<10^{4}$, the magnitude converges to unity for all $\kappa$. The phase velocity recovers the inviscid result (1.2) in its magnitude. The phase of $c$ also recovers the inviscid result (1.2) for frequencies $\Omega>10^{4}$. The horizontal oscillation of the waves follows the motion of the centre of mass in the high frequency regime. This 


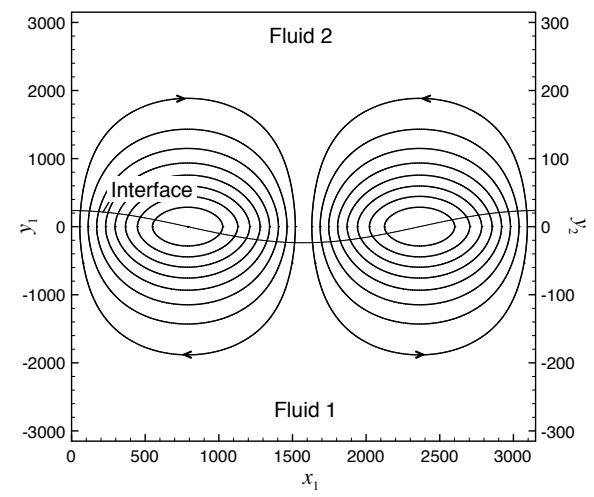

(a)

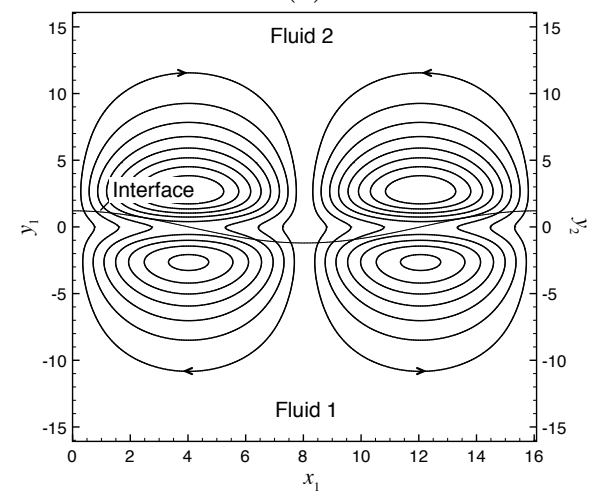

(c)

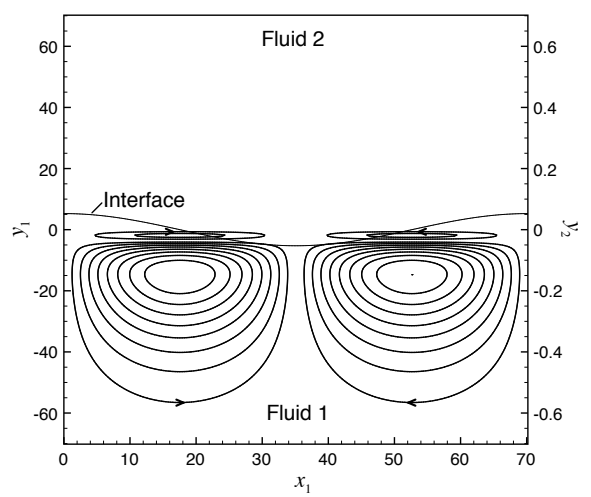

(b)

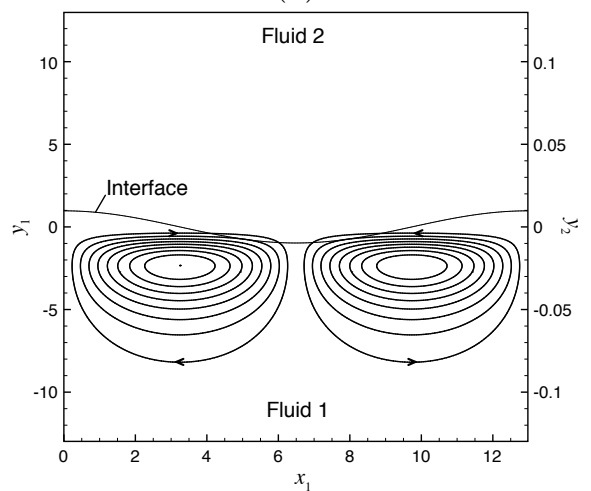

(d)

FIGURE 6. Streamlines of time-averaged flow for different oscillation frequencies and viscosity contrast values: (a) high frequency $\left(\kappa=10, \Omega=10^{5}, \Delta \psi=0.012\right)$, (b) moderate frequency $(\kappa=100, \Omega=0.5, \Delta \psi=6.2)$, (c) low frequency with a weak viscosity contrast $(\kappa=1, \Omega=100$, $\Delta \psi=0.023)(\mathrm{d})$ low frequency with a large viscosity contrast $(\kappa=100, \Omega=0.01, \Delta \psi=310)$ The density contrast $\rho$ is 0.9 for all of the figures.

behaviour confirms that the high frequency regime is the inviscid limit of the present stability problem.

As seen in equation (3.31), the time-averaged flow is directly related to the interface net evolution. The pattern of the flow is shown in figures 6 (a-d) for different oscillation frequencies and different viscosity contrasts. In each figure, the time-averaged shape of the interface wave is also illustrated. The control parameter B is set to be slightly larger ( 5 percent) than the critical value $\mathrm{B}_{c r}$, and the wavenumber is equal to the critical one $q_{c r}$.

For high frequency flow $\left(\Omega>10^{4}\right)$, the averaged flow consists of two cells per wavelength, as shown in (a). In these cells, there is no flow recirculation. All the streamlines pass through the interface. The whole time-averaged flow is associated with net growing motion of interface disturbance. Only the characteristic length seen in the cells is the wavelength. This indicates that vorticity is insignificant $(\triangle \psi \approx 0)$ in the bulk of the flow. Because these are characteristics of inviscid flow, the high frequency regime is really the inviscid limit of the problem.

For smaller frequencies, two or four cells are found per wavelength in the averaged flows, as seen in (b), (c) and (d). Flow can recirculate in cells, implying the importance of a steady streaming component in the averaged flow. This steady streaming is an Eulerian 
mean flow generated in the oscillatory flows through inertia effects. It is not associated to the interface net growing motion. The recirculation cells closest to the interface have their centre at a distance of around twice the Stokes boundary layer thickness from the interface. This length scale, different from the horizontal extension of the cells, implies that vorticity concentrates in these cells attached to the interface. We will use this finding later in a discussion on the instability mechanism. In fluids with a small viscosity contrast, the steady streaming component is not dominant, as seen in (c) where half of the streamlines pass through the interface. Recirculation cells are only small parts of the time-averaged flow pattern. In contrast, in fluids with large $\kappa$, as in (b) and (d), the flow is concentrated in Fluid 1 and the steady streaming is its main component. In Fluid 2, all the streamlines are related with the interface net growing motion (they are too weak and below the figures' resolutions in (b) and (d)). As seen in equation (2.8), the basic flow is almost null in Fluid 2 so that inertia-induced streaming does not exist. The observed concentration of the flow suggests that the instability is due to flow in the less viscous fluid for large $\kappa$.

Steady streaming is generated from the first order unsteady flow, as seen in equations (3.20-3.26). The latter unsteady flow is induced from two different origins. Difference between the shear rates $\partial U_{1} / \partial y$ and $\partial U_{2} / \partial y$ at the interface level $(y=0)$ yields a jump in velocity on the perturbed interface. Perturbation flow is induced to satisfy velocity continuity at the interface, as seen in equation (3.10). Another origin is the shear continuity (3.12). The difference between $\rho_{1} \nu_{1} \partial^{2} U_{1} / \partial y^{2}$ and $\rho_{2} \nu_{2} \partial^{2} U_{2} / \partial y^{2}$ at the interface level leads to a jump in the shear on the perturbed interface, which should be corrected through perturbation flow. According to these different origins, different components can be distinguished in the growth rate $\sigma^{(2)}$. There are component $\sigma_{\text {velocity }}^{(2)}$ resulting from the unsteady flow induced by the velocity continuity and a component $\sigma_{\text {shear }}^{(2)}$ from the flow induced by the shear continuity. The component $\sigma_{-}^{(2)}$ due to gravity and capillarity completes the total growth rate: $\sigma^{(2)}=\sigma_{\text {velocity }}^{(2)}+\sigma_{\text {shear }}^{(2)}+\sigma_{-}^{(2)}$. The latter term is always negative and tends to stabilize the system. A similar distinction of velocity- and shear-induced destabilization is made in the study of steady thin layer flows (Smith 1990; Charru \& Hinch 2000).

Figure 7 shows these velocity- and shear- induced components for different viscosity contrasts $\kappa . \sigma_{\text {velocity }}^{(2)}$ and $\sigma_{\text {shear }}^{(2)}$ are computed and normalized by $\left|\sigma_{-}^{(2)}\right|$ at the instability onset $\left(\mathrm{B}=\mathrm{B}_{c r}, q=q_{c r}\right)$. For small $\kappa$, the shear-induced component is dominant. In particular, for fluids of identical dynamical viscosities, the velocity-induced component is null because the shear rate of the basic flow is continuous at the interface level. For large $\kappa$, the dominance between $\sigma_{\text {velocity }}^{(2)}$ and $\sigma_{\text {shear }}^{(2)}$ interchanges with the frequency. The former is dominant at high frequencies of $\Omega>10^{4}$, the latter is at frequencies $\Omega$ smaller than $10 \rho$. The transition between these two regimes occurs within $10 \rho<\Omega<10^{4}$.

This finding implies that the mechanism of instability at frequencies $\Omega<10 \rho$ is different from the mechanism in the high frequency regime, which is the classical KelvinHelmholtz instability. At small frequencies, flow structures of thickness $\delta_{1}\left(\delta_{2}\right)$ are developed in Fluid 1 (Fluid 2) on the interface, as seen in figure 6 (b-d). This indicates vorticity diffusion in the proximity of the interface. Considering the short wave instability of steady two-layer Couette flows (Hooper \& Boyd 1983), Hinch (1984) gave a simple explanation of the growth of interface disturbance due to velocity-induced perturbation flow. Vorticity induced at the perturbed interface is advected by the basic flow to yield out-of-phase component, which enhances the interface disturbance. A similar explanation would be applicable to the present instability at $\Omega<10 \rho$. According to his analysis, 


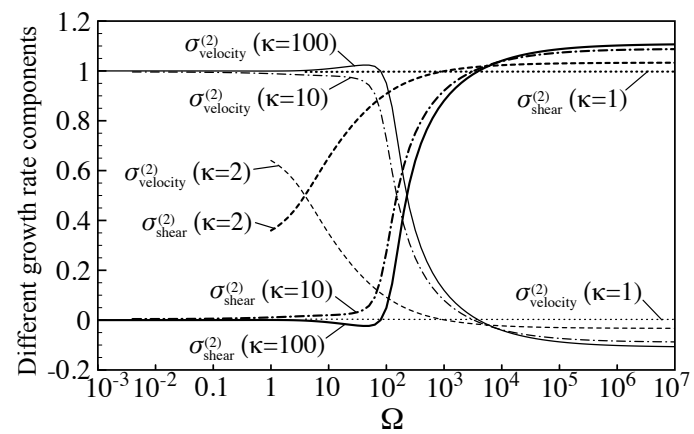

FiguRE 7. Different components of the third order growth rate $\sigma^{(2)}$ at the instability threshold. Both velocity- and shear-induced contributions, $\sigma_{\text {velocity }}^{(2)}$ and $\sigma_{\text {shear }}^{(2)}$, are shown for different viscosity contrasts $\kappa$ after normalization by the capillary-gravity contribution $\left|\sigma_{-}^{(2)}\right|$. ( $\rho=0.9$ for all the curves.)

the growth rate of disturbance can be estimated by $\sigma \sim O\left(\rho_{1} \alpha_{1}^{2} / k^{2} \rho_{2} \nu_{2}\right)$ for layers with a large viscosity contrast, where $\alpha_{1}$ is the shear rate in the less viscous layer. $k$ is the inverse of the extension of flow disturbance in the less viscous layer due to the vorticity diffusion, rather than the wavenumber (in unbounded Couette flows, both have the same order of magnitude). Application of this estimate to the present problem, with use of $\alpha_{1} \approx \Delta U / \delta_{1}$, gives $\sigma \sim O\left(\rho_{1} \Delta U^{2} / \rho_{2} \nu_{2}\right)$. The extension of vorticity diffusion layer, $k^{-1}$, has been estimated by $k^{-1} \approx \delta_{1}$, as it was indicated in the time-averaged flow patterns. On the other hand, according to the dispersion relation (4.2) obtained in the moderate frequency regime, the growth rate is scaled by $\sigma=(2 \pi K)^{2} \sigma^{(2)} \cdot \omega$ (the factor $\omega$ has been multiplied for the dimensional equation). It follows that $\sigma=2 \rho_{1} \Delta U^{2} / \rho_{2} \nu_{2}$. This agrees with the above estimate, indicating that the instability at small frequencies is due to the same mechanism as the short wave instability of the steady Couette flows.

As mentioned in section 2.1, the basic flow considered in the present paper can arise in thick fluid layers $\left(H_{1} \gg \delta_{1}, H_{2} \gg \delta_{2}\right)$ in a horizontally vibrated container and in a duct with a pulsating pressure gradient. As perturbation flow extends over a wavelength (see figure 6 (a), (c)), the thicknesses of the layers should also be larger than the wavelength for application of the present stability theory: $H_{1}>\lambda$ and $H_{2}>\lambda$. When the viscosity contrast is very large $(\kappa>100)$, however, the latter condition on $H_{2}$ can be omitted, since the flow concentrates only in the less viscous fluid (see figure 6 (b), (d)). The basic flow can also arise with fluids of large $\kappa$ in Couette configuration and in a rotated cylindrical tank. To apply the stability theory to these flows, the less viscous layer should be thicker than a wavelength for the same reason.

It is interesting to compare the present small amplitude theory with the numerical stability determination by Talib and her coworkers for finite-amplitude flows in a horizontally vibrated container. In figure 8 (a) and (b), the instability thresholds and most unstable wavenumbers reported in FIG. 11 of Talib et al. (2007) (hereafter referred to as Data I) and in FIG. 10 of Talib \& Juel (2007) (referred to as Data II) are shown, with the results of the small amplitude theory. Values of $\mathrm{B}_{c r}$ shown in (a) have been calculated from the reported critical amplitudes of container vibration, $U_{\text {cont }} / \omega\left(U_{\text {cont }}\right.$ is the container velocity), with use of the relationship (2.10). Data I is for fluid layers of thicknesses $H_{1}=H_{2}=20 \mathrm{~mm}$, vibrated at $20 \mathrm{~Hz}$. The physical properties of the fluids are $\rho_{1}=1752 \mathrm{~kg} / \mathrm{m}^{3}, \rho_{2}=966 \mathrm{~kg} / \mathrm{m}^{3}, \nu_{1}=1 \mathrm{~mm}^{2} / \mathrm{s}$ and $\gamma=7 \mathrm{mN} / \mathrm{m}$. These values 


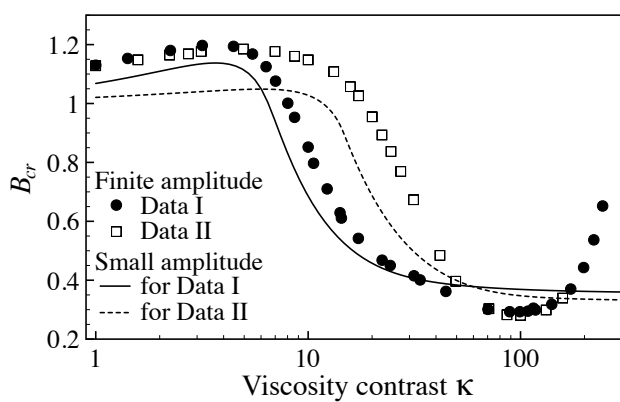

(a) Critical control parameter $\mathrm{B}_{c r}$

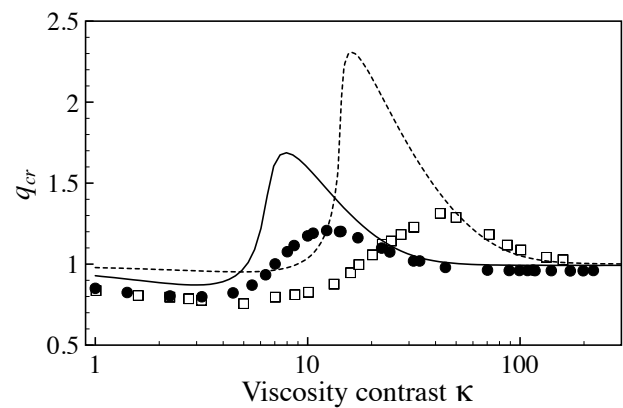

(b) Critical wavenumber $q_{c r}$

Figure 8. Comparisons with a finite-amplitude theory for the flow in a horizontally vibrated container. Markers shown as Data I are the results of Talib et al. (2007) for fluid layers of $\rho_{1}=1752 \mathrm{~kg} / \mathrm{m}^{3}, \rho_{2}=966 \mathrm{~kg} / \mathrm{m}^{3}, \gamma=7 \mathrm{mN} / \mathrm{m}, \nu_{1}=1 \mathrm{~mm}^{2} / \mathrm{s}$ and $H_{1}=H_{2}=20 \mathrm{~mm}$, vibrated at $\omega / 2 \pi=20 \mathrm{~Hz}$. Markers shown as Data II are the results of Talib \& Juel (2007) for such fluid layers that $\rho_{2} / \rho_{1}=0.490, \rho_{2} H_{2}^{3} \omega^{2} / \gamma=200, g / H_{2} \omega^{2}=0.199, H_{1} / H_{2}=1$ and $\omega H_{2}^{2} / \nu_{1}=39300$.

correspond to their experiments. The viscosity of the more viscous layer, $\nu_{2}=\kappa^{2} \nu_{1}$, is varied from $\kappa=1$ to $\kappa=245$. Data II is for fluid layers with a large interfacial tension. Dimensionless parameters for this data are given in legend of figure 8. If we chose the same $\rho_{1}, \nu_{1}, H_{1}$ and $H_{2}$ as Data I, these parameter values correspond to fluid layers of $\rho_{2}=859 \mathrm{~kg} / \mathrm{m}^{3}$ and $\gamma=210 \mathrm{mN} / \mathrm{m}$, vibrated at $6.29 \mathrm{~Hz}$. The viscosity $\nu_{2}=\kappa^{2} \nu_{1}$ is varied from $\kappa=1$ to $\kappa=160$. As seen in figure (a), the finite amplitude theory predicts non-monotonic behaviour of $\mathrm{B}_{c r}$ with $\kappa$ increasing. It increases slightly toward 1.2 at small $\kappa(\kappa<5)$, decreases toward 0.3 at intermediate $\kappa(5<\kappa<100)$ and increases again at large $\kappa(\kappa>100)$. The predictions of the small amplitude theory recover well this behaviour for both Data I and Data II, except the increase at large $\kappa$. This discrepancy is due to the fact that the more viscous layer is no longer thick compared with the Stokes boundary layer thickness $\delta_{2}$. Since the viscosity $\nu_{2}$ increases with $\kappa$, the Stokes boundary layer becomes thicker with $\kappa$ increasing and comparable with the layer thickness $H_{2}\left(\sim 1.5 \delta_{2}\right)$ at $\kappa=100$. The basic flow (2.5-2.8) will not approximate well the flow in the vibrated container. Both theories also predict non-monotonic behaviour of $q_{c r}$, as seen in figure (b). The small amplitude theory reproduces qualitatively the predictions of the finite amplitude theory over the whole $\kappa$ range, with excellent agreements at large $\kappa(\kappa>20$ for Data I, $\kappa>50$ for Data II). For both Data I and Data II, the predictions of the small amplitude theory do not agree quantitatively with the results of the finite amplitude theory. The small amplitude theory underestimates and overestimates the instability threshold at $\kappa \lesssim 40$ and at $40 \lesssim \kappa \lesssim 100$, respectively. It also overestimates $q_{c r}$ at $\kappa<20$ for Data I and $\kappa<50$ for Data II. These discrepancies will be due to the breaking of the small amplitude hypothesis. The Keulegan-Carpenter number $K$ is not small in the considered fluid configurations: $K>0.52$ for Data I and $K>0.75$ for Data II, according to the results of the finite amplitude theory. These values are beyond the validity limit of the small amplitude theory, which will be estimated later.

In figure 9 , the instability threshold and critical wavenumber predicted by the small amplitude theory are compared with carefully conducted experiments by Talib et al. (2007) in figure 9. They drove flows by horizontal vibration in a parallelepiped fluid container. Reported critical values of the vibration amplitude $U_{\text {cont }} / \omega$ of the container are transformed into the amplitude $\Delta U / \omega$ of the relative oscillatory motion between fluid layers by equation (2.10). In the figure, comparisons with the experiments reported in 


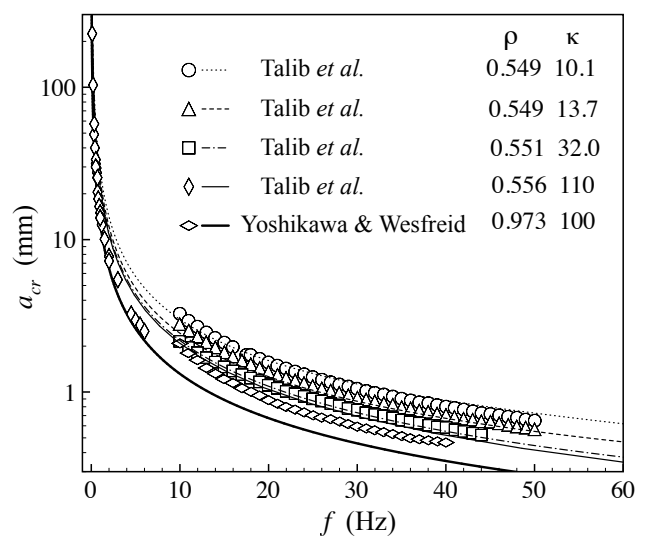

(a) Critical amplitude of the oscillation

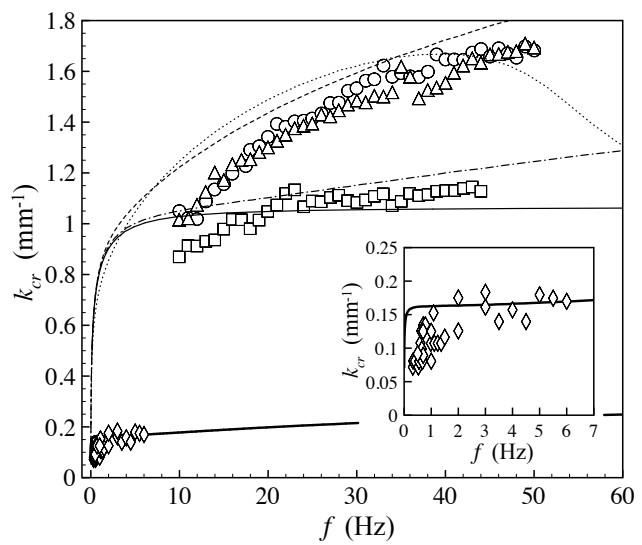

(b) Critical wavenumber

Figure 9. Comparisons with experiments of Talib et al. (2007) and Yoshikawa \& Wesfreid (2010). Physical properties of the less viscous liquids are $\rho_{1}=1752 \mathrm{~kg} / \mathrm{m}^{3}$ and $\nu_{1}=1.12 \mathrm{~mm}^{2} / \mathrm{s}$ with the interfacial tension $\gamma=6.8 \mathrm{mN} / \mathrm{m}$ for the former experiments and $1000 \mathrm{~kg} / \mathrm{m}^{3}$ and $1.0 \mathrm{~mm}^{2} / \mathrm{s}$ with $\gamma=10 \mathrm{mN} / \mathrm{m}$ for the latter. The density and viscosity contrasts are shown in the legend (experimental $k_{c r}$ are unavailable for $\kappa=110$ ).

our coupled paper are also included in order to show the applicability of the present theory to flows arising in another configuration. Flows were driven by alternate rotation of a cylindrical tank, as mentioned in section 1 .

In (a), comparisons show the theory correctly takes into account the influence of the density and viscosity contrasts to successfully predict the instability threshold. Excellent quantitative agreements for two experimental systems over the whole frequency and amplitude ranges $(0.1 \mathrm{~Hz}<\omega / 2 \pi<50 \mathrm{~Hz}, 0.6 \mathrm{~mm}<\Delta U / \omega<103 \mathrm{~mm})$, except for the experiments of Talib et al. (2007) with the largest viscosity contrast $(\kappa=110)$. This is due to the small thickness of the more viscous layer. The ratio $H_{2} / \delta_{2}$ is smaller than 1.9 for these experiments, while it is larger than 10.5, 7.7 and 3.3 for their experiments with $\kappa=10.1,13.7$ and 32.0, respectively. As discussed in section 2.1, the flow in a vibrated container is no longer approximated by the basic flow (2.5-2.8) when this ratio is small. In (b), comparisons show critical wavenumbers are also predicted well by the small amplitude theory. Experimentally observed viscosity and frequency dependence is correctly reproduced. However, comparisons are worse in experiments with smaller frequencies. This comes from the fact that the small amplitude hypothesis is less satisfied for them. Indeed, the finite amplitude theory of Talib et al. (2007) predicts excellently the critical wavenumber for their experiments over the entire frequency range. For the experiments presented in figure 9 , values of $K$ are larger than 0.5 when the frequency is smaller than $32 \mathrm{~Hz}, 24 \mathrm{~Hz}, 13 \mathrm{~Hz}$ and $1 \mathrm{~Hz}$ for experiments with viscosity contrasts $\kappa=10.1,13.7,32.0$ and 100, respectively. Below these frequencies, the small amplitude theory overestimates the critical wavenumber. This observation suggests that the present asymptotic theory is valid until a relatively large value of $K$. The validity limit would be around $K \approx 0.5$.

This validity limit can be transformed to a requirement on fluid properties. According to the definitions of the dimensionless parameters, the Keulegan-Carpenter number can be expressed as $K=\sqrt{2\left(\rho_{1}^{2}-\rho_{2}^{2}\right) g \lambda_{c a p}^{3} \mathrm{~B} q^{2} / \pi \rho_{1} \rho_{2} \nu_{2}^{2} \Omega^{2}}$. The validity condition $K \lesssim 0.5$ is rewritten with use of the latter equation:

$$
\frac{\left(\rho_{1}+\rho_{2}\right) \gamma^{3 / 2}}{\rho_{1} \rho_{2}\left(\rho_{1}-\rho_{2}\right)^{1 / 2} g^{1 / 2} \nu_{2}^{2}} \lesssim 0.25 \cdot \frac{\Omega^{2}}{16 \pi^{2} \mathrm{~B} q^{2}}
$$




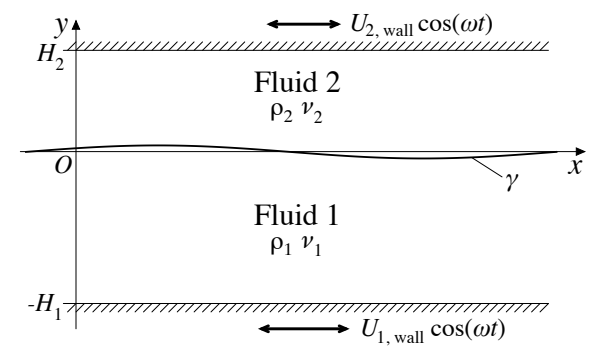

Figure 10. Two-layer oscillatory flows with bounding walls

This inequality, examined at the instability threshold for the most unstable mode, gives a requirement on fluid properties for the applicability of the present small amplitude theory. For realizing an experiment in the moderate frequency regime $\left(560 / \kappa^{2}<\Omega<10 \rho, \kappa>\right.$ $100)$ with fluids of $\rho_{1}=1752 \mathrm{~kg} / \mathrm{m}^{3}, \rho_{2}=966 \mathrm{~kg} / \mathrm{m}^{3}$ and $\gamma=7 \mathrm{mN} / \mathrm{m}$ as in the experiments of Talib et al. (2007), for example, the inequality (5.3) yields $\nu_{2} \gtrsim 1550 \Omega^{-1} \mathrm{~mm}^{2} / \mathrm{s}$. The critical parameters $\mathrm{B}_{c r}$ and $q_{c r}$ for the moderate frequency regime in table 1 have been invoked to derive this relationship. The requirement is therefore $\nu_{2} \gtrsim 280 \mathrm{~mm}^{2} / \mathrm{s}$ at the upper end of the moderate frequency regime $(\Omega=10 \rho=5.5)$. This inequality is normally satisfied, once we chose Fluid 2 under the condition $\kappa>100$. At a smaller frequency $\Omega=0.1$, however, Fluid 2 should be carefully chosen for $\nu_{2}$ to be larger than $15500 \mathrm{~mm}^{2} / \mathrm{s}$.

\section{Conclusion}

The linear stability of two-layer oscillatory flows was investigated for thick viscous fluid layers. The stability problem was formulated, respecting the dynamics inside the Stokes boundary layers developed on the interface in both layers. The problem was resolved for the asymptotic case of small $K$, i.e., a small oscillation amplitude compared with the wavelength. It was found that this asymptotic theory predicted a significant influence of the oscillation frequency and viscosity contrast on the instability threshold and the critical wavenumber, as reported in preceding experimental and theoretical works (Beysens et al. 1998; Ivanova et al. 2001a,b; Talib \& Juel 2007). Competition between different length scales $\left(\delta_{1}, \delta_{2}\right.$ and $\left.\lambda_{c a p}\right)$ was shown to accompany this influenced behaviour in the threshold and wavenumber. Different instability mechanisms were suggested: the same mechanism as in the classical Kelvin-Helmholtz instability for high frequency flows and the mechanism similar to the short wave instability in steady Couette flow for small frequency flows. Comparisons of the theoretical predictions with another theory and experiments in the literature justify the present asymptotic theory and enabled us to estimate the validity condition of the theory $(K \lesssim 0.5)$. For larger $K$, a theory for finite oscillation amplitude should be employed. A numerical resolution of the stability problem formulated in section 2 is performed and reported in the coupled paper for explaining experiments with large oscillation amplitudes (Yoshikawa \& Wesfreid 2010).

The authors would like to thank Dr. A. Juel and Prof. T. Mullin for fruitful discussions.

\section{Appendix A. Two-layer oscillatory flows in different configurations}

The generic configuration of (a) the flow in a horizontally vibrated container, (b) the duct flow generated by a pulsating pressure gradient and (c) the oscillatory Couette flow 
is illustrated in figure 10 . The wall velocities $U_{1 \text {,wall }}$ and $U_{2 \text {,wall }}$ are equal to the container velocity $U_{\text {cont }}$ for the flow (a) and null for the flow (b). The periodic solutions of the Navier-Stokes equations (2.1-2.2) are:

$$
\begin{aligned}
& U_{1}=\left(C_{1} e^{(1+i) \frac{y}{\delta_{1}}}+D_{1} e^{-(1+i) \frac{y}{\delta_{1}}}+E_{1}\right) e^{i \omega t}+c . c . \\
& U_{2}=\left(C_{2} e^{-(1+i) \frac{y}{\delta_{2}}}+D_{2} e^{(1+i) \frac{y}{\delta_{2}}}+E_{2}\right) e^{i \omega t}+c . c .
\end{aligned}
$$

The boundary conditions determining the constants $\left\{C_{1}, D_{1}, \ldots, E_{2}\right\}$ are those at the interface (2.4) and the no-slip conditions on the walls:

$$
U_{1}=U_{1, \text { wall }} \cos \omega t \quad \text { at } y=-H_{1} \quad \text { and } \quad U_{2}=U_{2, \text { wall }} \cos \omega t \quad \text { at } y=H_{2}
$$

Another condition completing the determination is specific for a flow configuration.

For the flow (a), the specific condition concerns the total volume flux. Due to the presence of the container endwalls, the flux should be equal to the volume swept by an endwall per unit time: $\int_{-H_{1}}^{0} U_{1} d y+\int_{0}^{H_{2}} U_{2} d y=\left(H_{1}+H_{2}\right) U_{\text {cont }} \cos \omega t$. Invoking this specific condition with the boundary conditions (2.4) and (A 2), one can calculate the constants to determine the flow (A 1). Analytical expressions of the solution can be found in Talib \& Juel (2007). For thick layers, neglecting corrections on the order of $O\left(\max \left(\frac{\delta_{1}}{H_{1}}, \frac{\delta_{2}}{H_{2}}\right)\right)$, one can derive the following approximative velocity fields:

$$
\left\{\begin{array}{l}
U_{1}=U_{\mathrm{i}}-\frac{\rho \kappa \Delta U}{1+\rho \kappa}\left[\cos \omega t-e^{\frac{y}{\delta_{1}}} \cos \left(\omega t+\frac{y}{\delta_{1}}\right)\right]+\frac{H_{2} \Delta U}{H_{1}+H_{2}} e^{-\frac{H_{1}+y}{\delta_{1}}} \cos \left(\omega t-\frac{H_{1}+y}{\delta_{1}}\right) \\
U_{2}=U_{\mathrm{i}}+\frac{\Delta U}{1+\rho \kappa}\left[\cos \omega t-e^{-\frac{y}{\delta_{2}}} \cos \left(\omega t-\frac{y}{\delta_{2}}\right)\right]-\frac{H_{1} \Delta U}{H_{1}+H_{2}} e^{-\frac{H_{2}-y}{\delta_{2}}} \cos \left(\omega t-\frac{H_{2}-y}{\delta_{2}}\right)
\end{array}\right.
$$

where $U_{\mathrm{i}}$ and $\Delta U$ are those given by equation (2.10). The third terms in the right-handsides are flow component generated by shear at the wall and negligible near the interface. The flow (A 3) is identical to the basic flow (2.5-2.8) considered in the present paper.

For the flow (b), the specific condition concerns the imposed pressure gradient $\Delta P \sin \omega t$ (per unit length): $\partial P_{1} / \partial x=\partial P_{2} / \partial x=\Delta P \sin \omega t$. Making use of this condition as well as the boundary conditions (2.4) and (A 2), one can determine the flow (A 1). For thick layers, the flow is given by the same expressions as (A 3), but with $U_{\mathrm{i}}$ and $\Delta U$ given by equation (2.11) and with different third terms in the right-hand-sides (the coefficients $\frac{H_{2} \Delta U}{H_{1}+H_{2}}$ and $\frac{H_{1} \Delta U}{H_{1}+H_{2}}$ are replaced by $-\frac{\rho \Delta U}{1-\rho}$ and $\frac{\Delta U}{1-\rho}$, respectively). Near the interface, this flow is identical to the considered basic flow.

For the flow (c), the specific condition is zero-pressure gradient $\partial P_{1} / \partial x=\partial P_{2} / \partial x=0$. Such flows can be realised in a vertically installed cylindrical Hele-Shaw cell with shearing top and/or bottom walls. When both walls have the same velocity, this flow configuration is equivalent to "co-flowing layer" considered in Talib (2006). Assuming a large viscosity contrast as well as $H_{1} \gg \delta_{1}$ and $H_{2} \ll \delta_{2}$, one can derive the following approximative expressions of the velocity fields:

$$
\left\{\begin{array}{l}
U_{1}=-U_{2, \text { wall }} e^{\frac{y}{\delta_{1}}} \cos \left(\omega t+\frac{y}{\delta_{1}}\right)+U_{1, \text { wall }} e^{-\frac{H_{1}+y}{\delta_{1}}} \cos \left(\omega t-\frac{H_{1}+y}{\delta_{1}}\right) \\
U_{2}=U_{2, \text { wall }} \cos \omega t
\end{array}\right.
$$

In the neighbourhood of the interface, this is identical to the considered basic flow (in the limit of large $\kappa$ ), as mentioned in section 2.1.

\section{Appendix B. Matrices}

Explicit definitions of the matrices and vectors used in the present paper are given here. The functions appearing in these definitions, $\varphi_{j}$ and $\widetilde{W}_{j, \pm 1}(j=1,2)$, mean their 
values at the interface level $(y=0)$.

$$
\begin{aligned}
& \boldsymbol{L}_{1}=\left[\begin{array}{cccc}
\frac{2 \pi q}{\kappa \sqrt{\Omega}} & \sqrt{\frac{4 \pi^{2} q^{2}}{\kappa^{2} \Omega}+i 2} & \frac{2 \pi q}{\sqrt{\Omega}} & \sqrt{\frac{4 \pi^{2} q^{2}}{\Omega}+i 2} \\
1 & 1 & -\kappa & -\kappa \\
\frac{8 \pi^{2} q^{2}}{\kappa^{2} \Omega} & \frac{8 \pi^{2} q^{2}}{\kappa^{2} \Omega}+i 2 & -\rho \kappa \frac{8 \pi^{2} q^{2}}{\Omega} & -\rho \kappa\left(\frac{8 \pi^{2} q^{2}}{\Omega}+i 2\right) \\
-\frac{2 \pi q}{\kappa \sqrt{\Omega}}\left(\frac{8 \pi^{2} q^{2}}{\kappa^{2} \Omega}+i 2\right) & -\frac{8 \pi^{2} q^{2}}{\kappa^{2} \Omega} \sqrt{\frac{4 \pi^{2} q^{2}}{\kappa^{2} \Omega}+i 2} & -\rho \frac{2 \pi q}{\sqrt{\Omega}}\left(\frac{8 \pi^{2} q^{2}}{\Omega}+i 2\right) & -\rho \frac{8 \pi^{2} q^{2}}{\Omega} \sqrt{\frac{4 \pi^{2} q^{2}}{\Omega}+i 2}
\end{array}\right] \\
& \boldsymbol{d}_{1}^{(0)}=\left[\begin{array}{c}
-\frac{\rho \kappa^{2}-1}{\rho \kappa^{2}} \widetilde{W}_{1,1}^{\prime} \\
0 \\
-\frac{\kappa+1}{\kappa} \widetilde{W}_{1,1}^{\prime \prime} \\
0
\end{array}\right] \\
& \boldsymbol{L}_{0}=\left[\begin{array}{cccc}
\frac{2 \pi q}{\kappa \sqrt{\Omega}} & \frac{2 \pi q}{\kappa \sqrt{\Omega}} & \frac{2 \pi q}{\sqrt{\Omega}} & -\frac{2 \pi q}{\sqrt{\Omega}} \\
1 & 0 & -\kappa & 0 \\
\frac{8 \pi^{2} q^{2}}{\kappa^{2} \Omega} & \frac{8 \pi^{2} q^{2}}{\kappa^{2} \Omega} & -\rho \kappa \frac{8 \pi^{2} q^{2}}{\Omega} & \rho \kappa \frac{8 \pi^{2} q^{2}}{\Omega} \\
-\frac{16 \pi^{3} q^{3}}{\kappa^{3} \Omega^{3 / 2}} & 0 & -\rho \frac{16 \pi^{3} q^{3}}{\Omega^{3 / 2}} & 0
\end{array}\right] \\
& \boldsymbol{d}_{0}^{(1)}=\left[\begin{array}{c}
-\varphi_{1}^{\prime}+\varphi_{2}^{\prime}+\frac{\rho \kappa^{2}-1}{2 \rho \kappa^{2}}\left[\widetilde{W}_{1,-1}^{\prime}\left(a_{1,1}^{(0)}+b_{1,1}^{(0)}\right)-c . c .\right] \\
-\varphi_{1}+\kappa \varphi_{2} \\
-\varphi_{1}^{\prime \prime}-\frac{4 \pi^{2} q^{2}}{\kappa^{2} \Omega} \varphi_{1}+\rho \kappa\left(\varphi_{2}^{\prime \prime}+\frac{4 \pi^{2} q^{2}}{\Omega} \varphi_{2}\right)+\frac{\kappa+1}{2 \kappa}\left[\widetilde{W}_{1,-1}^{\prime \prime}\left(a_{1,1}^{(0)}+b_{1,1}^{(0)}\right)-c . c .\right] \\
-Q_{1}+\rho Q_{2}-i \frac{\pi \rho\left(1+q^{2}\right)}{2 \kappa(1+\rho) \mathrm{B} \sqrt{\Omega}}
\end{array}\right]
\end{aligned}
$$

with

$$
\left\{\begin{array}{l}
Q_{1}=\varphi_{1}^{\prime \prime \prime}-\frac{12 \pi^{2} q^{2}}{\kappa^{2} \Omega} \varphi_{1}^{\prime}+\left[i \widetilde{W}_{1,-1}^{\prime}\left(a_{1,1}^{(0)}+b_{1,1}^{(0)}\right)-c . c .\right. \\
Q_{2}=\varphi_{2}^{\prime \prime \prime}-\frac{12 \pi^{2} q^{2}}{\Omega} \varphi_{2}^{\prime}+\left[i \widetilde{W_{2,-1}^{\prime}}\left(a_{2,1}^{(0)}+b_{2,1}^{(0)}\right)-c . c .\right]
\end{array}\right.
$$

\section{Appendix C. Symmetry}

Due to the left-right symmetry of the considered system, the time-periodic part of a velocity field at a given time is the mirror image of that after half a period with respect to the $y$ axis. The time periodic parts of the perturbed flows should therefore satisfy the following conditions:

$$
\widetilde{\psi}_{j,-m}=(-1)^{m-1} \widetilde{\psi}_{j, m}^{*} \quad(j=1,2)
$$

where the asterisk denotes the complex conjugate. Similar conditions are also imposed on the periodic interface disturbances:

$$
\widetilde{\eta}_{-m}=(-1)^{m} \widetilde{\eta}_{m}^{*}
$$

Appendix D. Coefficients $F_{j}, G_{j}$, and $H_{j}(j=1,2)$

$$
F_{1}=-\frac{\frac{\rho \kappa}{1+\rho \kappa}}{\left(\frac{2 \pi q}{\kappa \sqrt{\Omega}}+1-i\right)^{2}-\frac{4 \pi^{2} q^{2}}{\kappa^{2} \Omega}}
$$




$$
\begin{aligned}
G_{1} & =\frac{\rho \kappa}{i 2(1+\rho \kappa)} \\
H_{1} & =-\frac{\frac{2 \rho \kappa}{1+\rho \kappa}}{\left(\sqrt{\frac{4 \pi^{2} q^{2}}{\kappa^{2} \Omega}+i 2}+1-i\right)^{2}-\frac{4 \pi^{2} q^{2}}{\kappa^{2} \Omega}} \\
F_{2} & =\frac{\frac{1}{1+\rho \kappa}}{\left(\frac{2 \pi q}{\sqrt{\Omega}+1-i)^{2}-\frac{4 \pi^{2} q^{2}}{\Omega}}\right.} \\
G_{2} & =-\frac{1}{i 2(1+\rho \kappa)} \\
H_{2} & =\frac{\frac{2}{1+\rho \kappa}}{\left(\sqrt{\frac{4 \pi^{2} q^{2}}{\Omega}+i 2}+1-i\right)^{2}-\frac{4 \pi^{2} q^{2}}{\Omega}}
\end{aligned}
$$

\section{REFERENCES}

Benjamin, T. B. \& Ursell, F. 1954 The stability of a plane free surface of a liquid in vertical periodic motion. Proc. R. Soc. Lond. A 225, 505-515.

Beysens, D., Wunenburger, R., Сhabot, C. \& Garrabos, Y. 1998 Effect of oscillatory accelerations on two phase fluids. Microgravity Sci. Technol. 11 (3), 113-118.

Charru, François \& Hinch, E. John 2000 'Phase diagram' of interfacial instabilities in a two-layer Couette flow and mechanism of the long-wave instability. J. Fluid Mech. 414, $193-223$.

Coward, A. V. \& Papageorgiou, D. T. 1994 Stability of oscillatory two-phase Couette flow. IMA J. Appl. Math. 53, 75.

Davis, S. H. 1976 The stability of time-periodic flows. Annu. Rev. Fluid Mech. 8, 57-74.

HiNCH, E. 1984 A note on the mechanism of the instability at the interface between two shearing fluids. J. Fluid Mech. 144, 463-465.

Hogan, J. M. \& Ayyaswamy, P. S. 1985 Linear stability of a viscous-inviscid interface. Phys. Fluids 28 (9), 2709-2715.

Hooper, A. P. \& Boyd, W. G. C. 1983 Shear-flow instability at the interface between two viscous fluids. J. Fluid Mech. 128, 507-528.

Ivanova, A. A., Kozlov, V. G. \& Evesque, P. $2001 a$ Interface dynamics of immiscible fluids under horizontal vobration. Fluid Dynamics 36, 362.

Ivanova, A. A., Kozlov, V. G. \& Tachkinov, S.I. $2001 b$ Interface dynamics of immiscible fluids under circularly polarized vibration (experiment). Fluid Dynamics 36, 871.

KAmachi, Masafumi \& Honji, HiRoyuki 1982 The instability of viscous two-layer oscillatory flows. Journal of the Oceanographical Society of Japan 38, 346-356.

Kelly, R. E. 1965 The stability of an unsteady Kelvin-Helmholtz flow. J. Fluid Mech. 22, 547.

Khenner, M. V., Lyubimov, D. V., Belozerova, T. S. \& Roux, B. 1999 Stability of planeparallel vibrational flow in a two layer system. Eur. J. Mech. B 18, 1085.

King, M. R., Leighton, D. T. \& MCCready, M. J. 1999 Stability of oscillatory two phase Couette flow : Theory and experiment. Phys. Fluids 11, 833.

Kumar, K. \& Tuckerman, L. S. 1994 Parametric instability of the interface between two fluids. J. Fluid Mech. 279, 49-68.

Legendre, Marc, Petitjeans, Philippe \& Kurowski, Pascal 2003 Instabilities at the interface between miscible fluids under a horizontal oscillating forcing. Comptes Rendus Mécanique 331 (9), 617-622.

Lindsay, K. A. 1984 The Kelvin-Helmholtz instability for a viscous interface. Acta Mechanica 52, 51-61. 
Lyubimov, D. V. \& Cherepanov, A. A. 1987 Development of a steady relief at the interface of fluids. Fluid. Dyn. Res. 22, 849.

Lyubimov, D. V., Lyubimova, T. P. \& Cherepanov, A. A. 2003 Dynamics of interfaces in vibration fields (in Russian). Moscow: FizMatLit.

Miles, John \& Henderson, Diane 1990 Parametrically forced surface waves. Annu. Rev. Fluid Mech. 22, 143-165.

OR, A. C. 1997 Finite-wavelength instability in a horizontal liquid layer on an oscillting plane. J. Fluid Mech. 335, 213-232.

Rousseaux, Germain, Yoshikawa, Harunori, Stegner, Alexandre \& Wesfreid, José EduARdo 2004 Dynamics of transient eddy above rolling-grain ripples. Phys. Fluids 16 (4), 1049-1058.

Shyн, C. K. \& Munson, B. R. 1986 Interfacial instability of an oscillating shear layer. J. Fluid Eng. 108, 89-92.

Smith, M. K. 1990 The mechanism for the long-wave instability in thin liquid films. J. Fluid Mech. 27, 469-485.

TALIB, E. 2006 Instability of oscillatory two-layer viscous flow. PhD thesis, University of Manchester.

Talib, Emma, Jalikop, Shreyas V. \& Juel, Anne 2007 The influence of viscosity on the frozen wave instability: theory and experiment. J. Fluid Mech. 584, 45-68.

Talib, Emma \& Juel, Anne 2007 Instability of a viscous interface under horizontal oscillation. Phys. Fluids 19, 092102.

Wolf, G. H. 1969 The dynamic stabilization of the Rayleigh-Taylor instability and the corresponding dynamic equilibrium. Z. Physik 227, 291-300.

Yıн, C.-S. 1967 Instability due to viscosity stratification. J. Fluid Mech. 26, 337.

Yoshikawa, Harunori NAKagawa 2006 Instabilités des interfaces sous oscillations. PhD thesis, Université Paris 6, Paris.

Yoshikawa, H. N. \& Wesfreid, J. E. 2010 Oscillatory Kelvin-Helmholtz instability: Part 2. An experiment in fluids with a large viscosity contrast. J. Fluid Mech. (in press). 